\title{
1 Evolution of phenotypic variance provides insights into the genetic basis of
}

\section{2 adaption}

\section{Author list:}

4 Wei-Yun Lai ${ }^{1,2}$, Viola Nolte ${ }^{1}$, Ana Marija Jakšić ${ }^{1,2,3}$ and Christian Schlötterer ${ }^{*}$

$5 \quad{ }^{1}$ Institut für Populationsgenetik, Vetmeduni Vienna, Vienna, Austria.

$6 \quad{ }^{2}$ Vienna Graduate School of Population Genetics, Vetmeduni Vienna, Vienna, Austria.

$7 \quad{ }^{3}$ Current affiliation: École polytechnique fédérale de Lausanne, Lausanne, Switzerland

$8 \quad{ }^{*}$ Correspondence: christian.schloetterer@vetmeduni.ac.at; Tel.: +43-1-25077-4300.

10 Keywords:

11 Phenotypic variance, temperature adaptation, Drosophila simulans, experimental evolution 


\section{Abstract}

13 Most traits are polygenic and the contributing loci can be identified by GWAS. Their

14 adaptive architecture is, however, poorly characterized. Here, we propose a new approach to

15 study the adaptive architecture, which does not depend on genomic data. Relying on

16 experimental evolution we measure the phenotypic variance in replicated populations during

17 adaptation to a new environment. Extensive computer simulations show that the evolution of

18 phenotypic variance in a replicated experimental evolution setting is a powerful approach to

19 distinguish between oligogenic and polygenic adaptive architectures. We apply this new

20 method to gene expression variance in male Drosophila simulans before and after 100

21 generations of adaptation to a novel hot environment. The variance change in gene expression was indistinguishable for genes with and without a significant change in mean expression after 100 generations of evolution. We conclude that adaptive gene expression

24 evolution is best explained by a highly polygenic adaptive architecture. We propose that the

25 evolution of phenotypic variance provides a powerful approach to characterize the adaptive

26 architecture, in particular when combined with genomic data. 


\section{Introduction}

29 It is widely accepted that most complex traits have a polygenic or even infinitesimal basis

30 (Ayroles et al., 2009; Boyle et al., 2017; Liu et al., 2019). Nevertheless, it is difficult to

31 predict which of these loci are responding to selection when a population is exposed to a new

32 selection regime. If pleiotropic constraints are strong, only a small subset of the genes constituting are free to respond to respond to selection. Hence, the genetic basis of the adaptive response of a complex trait (i.e. adaptive architecture (Barghi et al., 2020)) may differ substantially from the genetic architecture. Since even for large phenotypic changes the genetic basis of an adaptive response is difficult to study when more than a handful of genes are contributing, we introduce a new approach to study the complexity of the adaptive architecture. Rather than aiming to map the contributing loci, we propose to study the evolution of phenotypic variance in an experimental evolution framework.

The phenotypic variance of a quantitative trait is a key determinant for its response to selection. It can be decomposed into genetic and environmental components (Falconer and Mackay, 1963). Over the past years, mathematical models have been developed which describe the expected genetic variance of a quantitative trait under selection and its maintenance in evolving populations (Bulmer, 1972; Chevalet, 1994; Kimura and Crow, 1964; Turelli, 1984). For infinitely large populations and traits controlled by many independent loci with infinitesimal small effect, changes in trait optimum are not expected to affect the phenotypic variance (Lande, 1976). A much more complex picture is expected when the effect sizes are not equal, the population size is finite, or the traits have a simpler 50 genetic basis (Barton and Keightley, 2002; Barton and Turelli, 1987; Franssen et al., 2017; 51 Hayward and Sella, 2019; Jain and Stephan, 2015; Keightley and Hill, 1989). For instance, for a trait with oligogenic architectures, the genetic variance could drop dramatically during 
adaptation, while with polygenic architectures, only minor effects on the variance are expected (Barton et al., 2017; Franssen et al., 2017; Jain and Stephan , 2015). These studies suggest that a time-resolved analysis of phenotypic variance has the potential to shed light onto the complexity of the underlying adaptive architecture.

Despite its potential importance for the understanding of adaption, we are faced with the situation that very few empirical data are available for the evolution of phenotypic variance. The use of natural populations to study changes in phenotypes and even more so phenotypic variances is limited, as the environmental heterogeneity cannot be controlled. A complementary approach to study the evolution of phenotypic variance is experimental evolution (Kawecki et al., 2012). With replicated populations starting from the same founders and evolving under tightly controlled environmental conditions, experimental evolution provides an enormous potential to study the evolution of phenotypic variance.

Most experimental evolution studies in sexual populations focused on the evolution of phenotypic means, rather than variance (Burke et al., 2010; Chippindale et al., 1996; Jakšić et al., 2020; Mallard et al., 2018). A notable exception is a study which applied fluctuating, stabilizing and disruptive selection to a small number of traits (wing shape) and observed a change of the phenotypic variance (Pélabon et al., 2010). Instead of looking at a preselected subset of phenotypes which limits the generality, we will focus on gene expression, a set of molecular phenotypes, which can be easily quantified since microarrays and, more recently, RNA-Seq have become available. Importantly, the expression levels of genes exhibit the same properties (e.g.: continuality and normality) as other complex quantitative traits (Mackay et al., 2009). Thus, gene expression has also been widely employed to search for putative adaptive traits of locally adapted populations (Romero et al., 2012; Signor and 
78 Nuzhdin, 2018; Sork, 2017) or ancestral and evolved populations in the context of experimental evolution (Ferea et al., 1999; Huang and Agrawal, 2016; Lenski et al., 1994;

$80 \quad$ Mallard et al., 2018).

81

In this study, we used forward simulations that match not only essential design features of typical experimental evolution studies, but also incorporate realistic parameters of the genetic architecture. We recapitulate the classic expectations that even a moderately polygenic architecture is associated with a high stability of the phenotypic variance of a selected trait across different phases of adaptation. Applying this insight to a real dataset (Barghi et al., 2019; Hsu et al., 2020; Jakšić et al., 2020), we show that a considerable set of genes changed their mean expression, but their expression variance was indistinguishable from genes without changes in mean expression. We propose that this pattern reflects that adaptive gene expression evolution generally has a polygenic basis.

\section{Results}

93 The central idea of this study is that the complexity of an adaptive trait can be estimated from

94 the trajectory of the phenotypic variance during adaptation: the phenotypic variance remains relative stable for a trait with polygenic (infinitesimal) architecture while it changes across generations for a trait with oligogenic architecture. Although this prediction has been

97 illustrated in multiple theoretical and simulation studies (e.g.: Barton et al., 2017; Franssen et 98 al., 2017; Jain and Stephan, 2015), as the first step of this study, we explored to what extent it can be generalized to a typical E\&R setting considering a broader parameter space. Assuming additivity and a negative correlation between ancestral allele frequency and effect size (Otte et al., 2020) (Figure 1b), we simulated traits adapting to a mild/distant shift in trait optimum

102 with weak/intermediate/strong stabilizing selection (Figure 1a and Figure 1 - Figure 
supplement 1). With three different distributions of effect size (Figure 1c) we investigate how the number of contributing loci affects the phenotypic variance.

106 We monitored the change in phenotypic variance over 200 generations, which was sufficient 107 to reach the trait optimum for most parameter combinations (Figure 2 - Figure supplement 4 and 5). We compared the change in variance relative to the start of the experiment in populations with and without selection. First, we studied a mild (one standard deviation of

110 the ancestral phenotypic distribution) shift in trait optimum (Figure 2 and Figure 2 - Figure

111 supplement 2). As expected for a founder population derived from a substantially larger

112 natural population, we find that even under neutrality the phenotypic variance does not remain constant, but gradually decreases during 200 generations of experimental evolution

114 (Figure 2). This loss of variance is best explained by the fixation of variants segregating in

115 the founder population and we did not simulate new mutations, as they do not contribute to 116 adaptation in such short time scales (Burke et al., 2010). Our simulations show that even 117 experimental evolution studies with moderate population sizes and linkage very nicely recapitulate the patterns from previous studies (Barton et al., 2017; Franssen et al., 2017; Jain

119 and Stephan , 2015). A pronounced drop in phenotypic variance is observed while a trait is approaching a shifted optimum with few contributing loci (Figure 2). When more loci are contributing to the selected phenotype, the difference to neutrality becomes very small (Figure 2 and Figure 2 - Figure supplement 2). In addition to the number of contributing loci,

123 also the heterogeneity in effect size among loci and the shape of the fitness function have a 124 major impact. The larger the difference in effect size is, the more pronounced was the influence of the number of contributing loci (Figure 2). The opposite effect was seen for the width of the fitness function - a larger variance decreased the influence of the number of

127 contributing loci (Figure 2). Importantly, these patterns were not affected by the duration of 
the experiment-qualitatively identical patterns were seen at different time points until generation 200 .

131 For a more distant trait optimum (three standard deviations of the ancestral phenotypic

132 distribution), we noticed some interesting dynamics that were not apparent for a closer trait optimum (Figure 2 - Figure supplement 1 and 3). The most striking one was the temporal

134 heterogeneity of the phenotypic variance for few contributing loci of unequal effects. During

135 the early stage of adaptation, the variance increased and dropped later below the variance in

136 the founder population. With an increasing number of contributing loci, this pattern

137 disappeared and closely matched the neutral case (Figure 2 - Figure supplement 1).

138 Modifying dominance did not change the overall patterns-with a large number of contributing

139 loci the variance fitted the neutral pattern best (Figure 2 - Figure supplement 6). Overall, our

140 simulations indicate that only for a small set of parameters, the variance will increase during

141 the early stage of adaptation - in particular scenarios based on a few contributing loci with

142 very different effect sizes. The large influence of key parameters of the adaptive architecture,

143 in particular the number of contributing loci and their effect sizes on the temporal phenotypic

144 variance dynamics, suggest that it should be possible to exploit this for a test of polygenic

145 adaptation, which is independent from genomic data.

147 As a proof of principle, we studied the evolution of gene expression variance in replicated 148 populations evolving in a new hot temperature regime (Barghi et al., 2019; Hsu et al., 2020;

149 Jakšić et al., 2020). The evolved populations were derived from the same ancestral 150 population, but evolved independently for 100 generations in a novel temperature regime 151 with daily temperature fluctuations between 18 and $28^{\circ} \mathrm{C}$ (Figure 3a). Rather than relying on 152 pooled samples that allow only to estimate means, we quantified gene expression of 
individuals from reconstituted ancestral populations and two evolved populations in a we estimated a heritability of around $60 \%$ across the transcriptome among individual flies

156 from different families, which demonstrates the robustness of our experimental setup to

157 estimate expression variances (Method - Figure supplement 1; See Materials and methods).

158 Principle Component Analysis (PCA) indicated that $11.9 \%$ of the variation in gene expression can be explained by the first PC which separates evolved and ancestral populations, reflecting clear adaptive gene expression changes in response to the novel, hot temperature regime (Figure 3b). The means and variances of the expression of each gene were estimated and compared between the reconstituted ancestral populations and the two evolved populations (Method - Figure supplement 2 and 3; See Materials and methods). Due to the usage of different lot numbers for the RNA-Seq library preparation (Supplementary file 1), we only contrasted ancestral and evolved samples generated with the same lot number (See Materials and methods) to avoid any unnecessary confounding results.

168 The comparison of ancestral and evolved populations identified 2,812 genes in the first replicate and 2,704 genes in the second replicate which significantly changed mean expression in the evolved flies (FDR $<0.05$, Supplementary file 2 ). With about $20 \%$ of the genes changing mean expression, it is apparent that both populations evolved during 100 generations of exposure to a novel environment. $93.8 \%$ of the genes with a significant mean expression change in both populations changed in the same direction, more than expected by

174 chance (Figure 4a, $\chi^{2}=896.34$, p-value $<2.2 \times 10^{-16}$ ). This concordance suggests that most of the altered expression means are mainly driven by selection, rather than by drift. We

176 quantified the expression change by relating the change in gene expression to the standard 177 deviation in the ancestral population. The differentially expressed genes in both replicates 
178 showed a broad distribution of expression change, but the mean expression changed by one

179 standard deviation (Figure 4b). Assuming that all expression phenotypes reached trait

180 optimum, this reflects on average a moderate shift in trait optimum.

182 Consistent with computer simulations, the analysis of gene expression variance showed a slight decrease in evolved populations relative to the ancestral ones (median F-value $=0.84$ in both replicates). Only a small subset of gene ( $\mathrm{n}=125$ and 97 in each replicate) experienced a substantial reduction in variance. Because this reduction in variance is probably driven by a different evolutionary force, we discuss them elsewhere (Lai et al., 2021). For the remaining genes, we related the changes in gene expression variance of selected genes to non-selected ones, we tested whether the variance changes in expression differ between the genes with significant mean expression changes and those without. Hence, we assume that genes with significant mean expression changes are under selection and the rest of the transcriptome has no large effect on the fitness (neutral). Remarkably, the changes in variance of putative adaptive genes with significant mean expression changes are indistinguishable from the genes that do not change their mean expression (Figure 4c). This suggests that the selection on mean expression is independent from the change in variance during adaptation in the focal populations. While variance estimates from two time points do not provide sufficient power to estimate the number of contributing loci in absence of more information about the adaptive architecture, our computer simulations (Figure 2), suggest in line with previous theoretical work (Barton et al., 2017; Jain and Stephan, 2015) that the observed stability in variance evolution reflects a polygenic architecture underlying the adaptive gene expression evolution.

201 Since we only explored two time points rather than a full time series, it may be possible that 202 an oligogenic basis could also result in a similar phenotypic variance change as a polygenic 
203 architecture (Figure 2 - Figure supplement 1 and 3). This can be seen in an intuitive case

204 when a single/few major effect allele(s) starts at a low frequency and becomes fixed (Yoo et

205 al., 1980). Because an oligogenic basis results in a highly parallel genomic selection response

206 (Figure 2 - Figure supplement 7), it is possible to distinguish polygenic and oligogenic

207 architectures with phenotypes from two time points only, when genomic data are available.

208 Because the genomic signature in the same experiment uncovered a highly heterogeneous

209 selection response (Barghi et al., 2019), we can exclude the unlikely explanation of an

210 oligogenic architecture resulting in a similar expression variance as non-selected genes.

211 Rather, we conclude that the adaptive response in gene expression is best explained by a

212 highly polygenic architecture. 


\section{Discussion}

214 Population genetics has a long tradition to characterize adaptation based on the genomic

215 signature of selected loci (Nielsen, 2005). Nevertheless, for selected phenotypes with a

216 polygenic architecture, the contribution of individual loci to phenotypic change may be too

217 subtle to be detected with classic population genetic methods (Pritchard et al., 2010). Even

218 with an oligogenic basis, the identification of the selection targets with classic population

219 genetic tests can be challenging.

220

221 Here, we used a conceptually different approach, which does not build on the genomic

222 signature, to infer the adaptive architecture. Reasoning that experimental evolution is probably the best approach to obtain phenotypic time series, we performed computer simulations specifically tailored to typical experimental evolution studies with Drosophila.

We showed that the temporal dynamics of the phenotypic variance is strongly affected by the number of contributing loci and other parameters of the adaptive architecture, such as the distribution of effect size and the underlying fitness function. Similar to the classic

228 Castle-Wright estimator (Castle, 1921) that estimates the number of loci contributing to a quantitative trait from the phenotypic variance of the F2, we propose that the temporal heterogeneity of the phenotypic variance can be used to estimate the number of loci contributing to the adaptive response of a phenotype as well as other parameters of the adaptive architecture. Hence, unlike other approaches to characterize polygenic adaptation, the proposed estimator does not require genetic data when phenotypic time series data are available.

236 Because gene expression changes are constituting a major component of adaptation to a novel

237 environment (Romero et al., 2012; Signor and Nuzhdin, 2018; Sork, 2017), it provides an 
excellent model to evaluate a variance-based test for polygenicity. Gene expression is modified by many trans-acting factors and some cis-regulatory variation. Adaptive gene expression changes can be either driven by polymorphism in cis-regulatory elements or by trans-acting variants. While interspecific differences in gene expression are predominantly caused by cis-regulation (Wittkopp et al., 2004), intraspecific variation is mostly driven by trans regulatory changes (Suvorov et al., 2013; Wittkopp et al., 2008). Adaptive gene expression changes which are well-characterized on the molecular level typically have a cis-regulatory basis that is not only frequently associated with the insertion of a transposable element (e.g.: (Daborn et al., 2002)) but also sometimes with multiple regulatory variants

247 (Endler et al., 2018). Two lines of evidence suggest that cis-regulatory variation cannot be the driver of adaptive gene expression changes observed in this study. First, the mutational target size is too small to harbor a sufficiently large number of alleles segregating in the founder population. Second, too few recombination events occur during the experiment to uncouple regulatory variants located on a given haplotype such that they could generate a signal of polygenic adaptation. More likely, the polygenic adaptive architecture of gene expression change reflects the joint effects of many trans-acting variants.

Because we could only analyze phenotypic data from two time points, the founder population and replicate populations evolved for 100 generations, we were not able to obtain a more quantitative estimate of the number of contributing loci, in particular as other parameters of

258 the adaptive architecture are not known and need to be co-estimated. For the distinction between an oligogenic and polygenic basis, we additionally relied on the heterogeneity of genomic selection signatures among replicates, because for some parameter combinations the oligogenic response can also result in a similar phenotypic variance as a polygenic one, but

262 with a much higher parallel response of genomic markers (Figure 2 - Figure supplement 7). 
263 Hence, not only more time points describing the phenotypic trajectory, but also some

264 genomic data could contribute to infer the adaptive architecture in experimental evolution

265 studies. The extension of this approach to natural populations faces several challenges. First,

266 phenotypic time series over evolutionary relevant time scales are rare (but see (Clutton-Brock

267 and Pemberton, 2004)) and second, the distinction of environmental heterogeneity from

268 genetic changes is considerably more challenging than under controlled laboratory

269 conditions.

270

271 


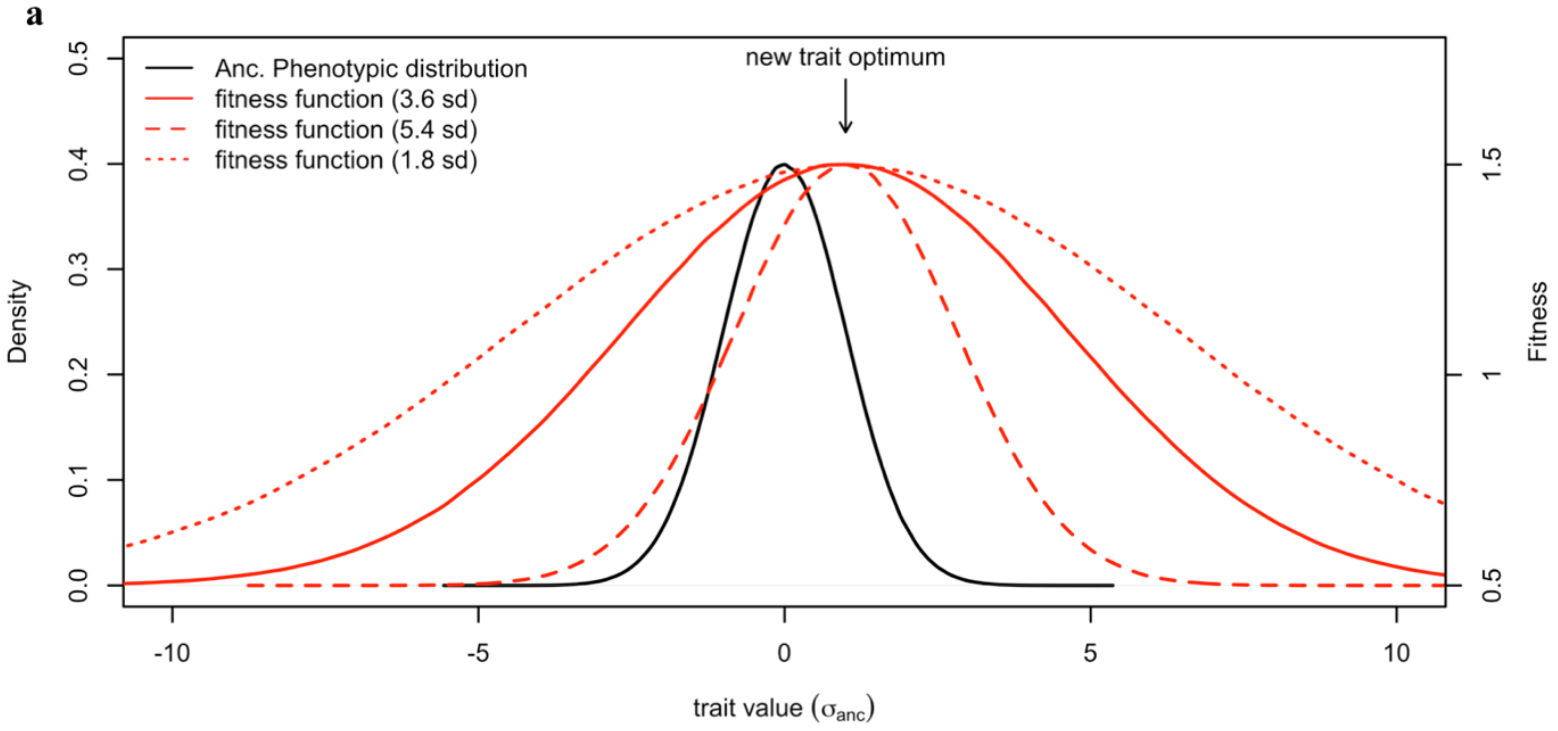

b

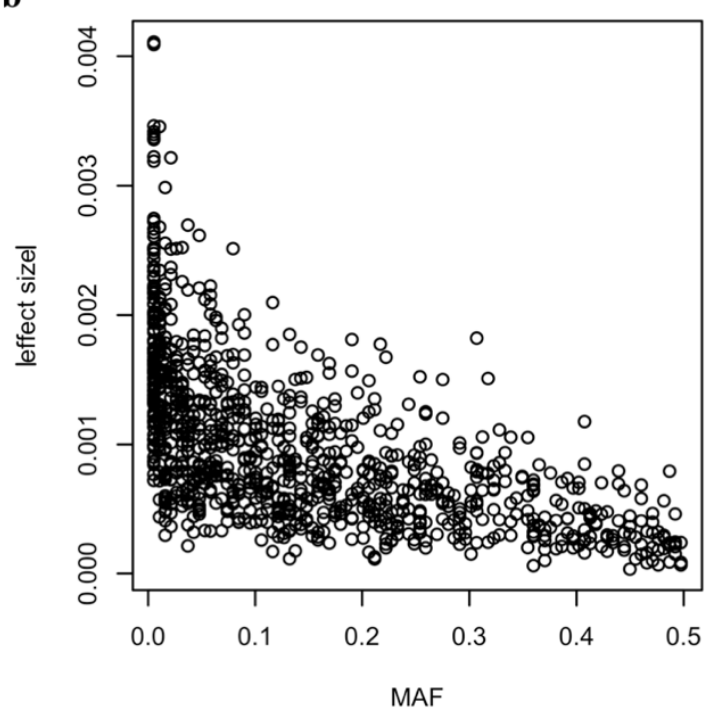

c

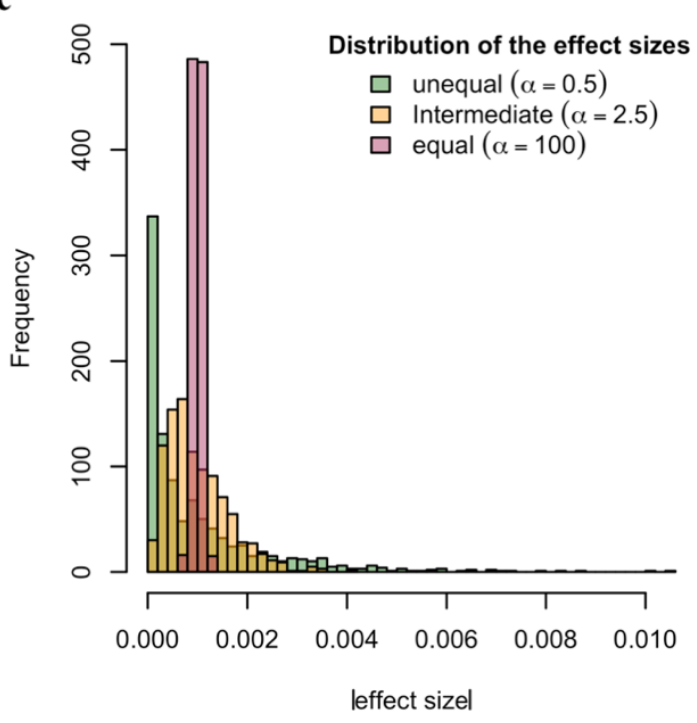

272

Figure 1. Simulating polygenic adaptation to a shift in trait optimum with different

parameter combinations. a. For the computer simulations we consider a quantitative trait (in black) experiencing a sudden shift in trait optimum under stabilizing selection. The underlying fitness functions are illustrated in red. The new trait optimum is shifted from the ancestral trait mean by one/three standard deviation of the ancestral trait distribution. The strength of stabilizing selection is modified by changing the variance of the fitness function: 1.8, 3.6 and 5.4 standard deviations of the ancestral trait distribution. b. The negative correlation between the allele frequencies and the effect sizes $(\mathrm{r}=-0.7$, estimated in Barghi et 
281 al., 2019). We consider such negative correlation when assigning the effect sizes to variants

282 underlying a simulated trait. c. The distribution of effect sizes of the contributing loci is

283 determined by the shape parameter $(\alpha)$ of gamma sampling process $(\alpha=0.5,2.5$ and 100$)$. 


\section{Mild optimum shift $\left(1 \sigma_{\text {anc }}\right)$}

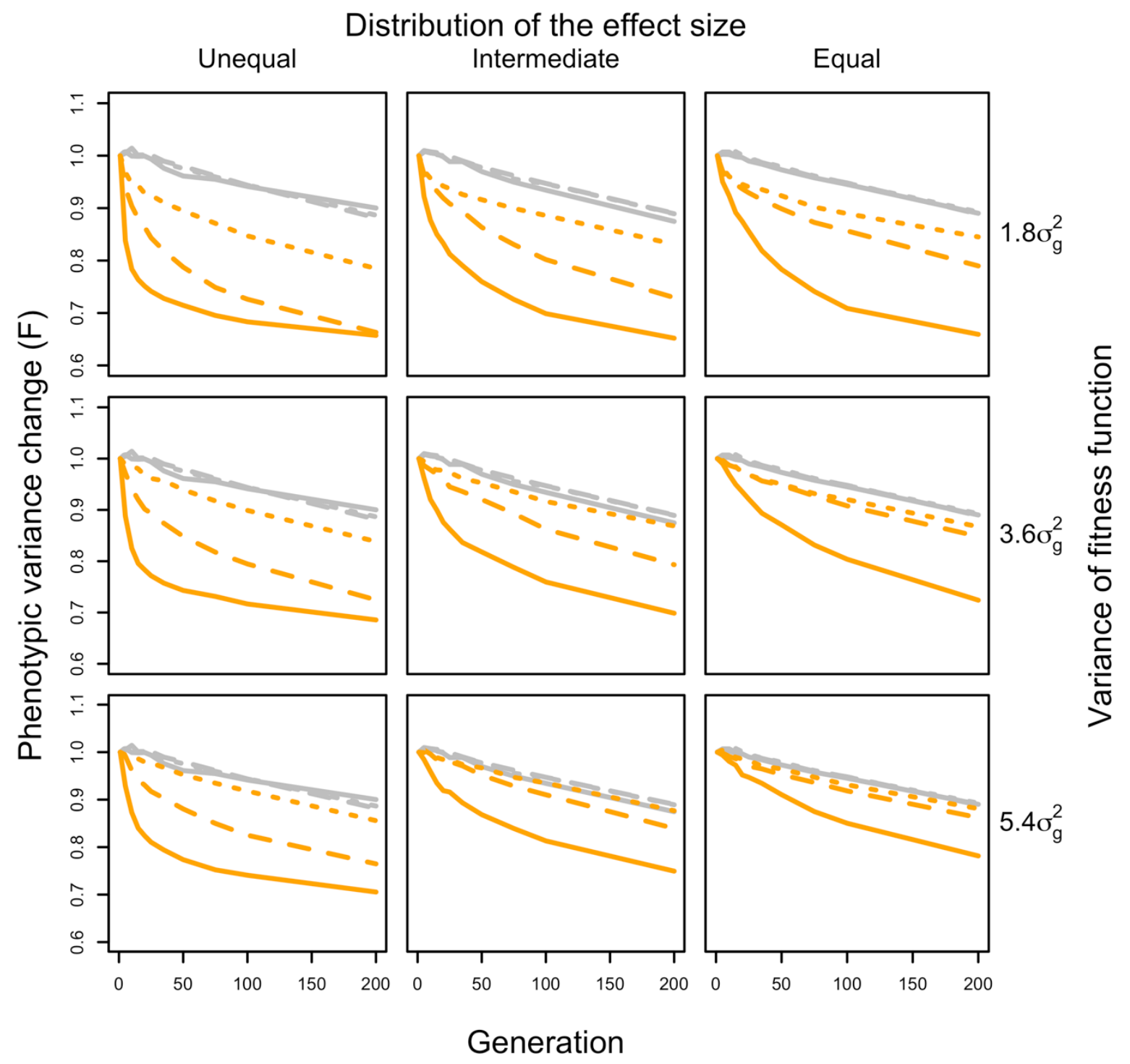

Figure 2. The trajectory of changes in phenotypic variance during adaptation to a mild

287 optimum shift. The changes in phenotypic variance within 200 generations adapting to a moderate optimum shift (orange) are compared to the changes under neutrality (grey) on y axis. The change in variance $(\mathrm{F})$ is calculated as the ratio of phenotypic variance between each evolved time point (generation $x$ ) and the ancestral state $\left(\sigma_{x}^{2} / \sigma_{0}^{2}\right)$. The simulations cover traits controlled by varying numbers of loci underlying the adaptation with three different

292 distributions of effect sizes (columns) under different strength of stabilizing selection (rows).

293 For each scenario, 1000 runs of simulations have been performed. Only traits with the most 
294 (dotted lines, 1000 loci), intermediate (dash lines, 50 loci) and the least (solid lines, 5 loci)

295 polygenic architectures are shown. In all scenarios, the variance of the trait decreases

296 drastically when the adaptation is controlled by a small number of loci (orange solid lines; 5

297 loci). While, for traits with extremely polygenic basis, the phenotypic variance stays stable

298 over time (orange dotted lines). 


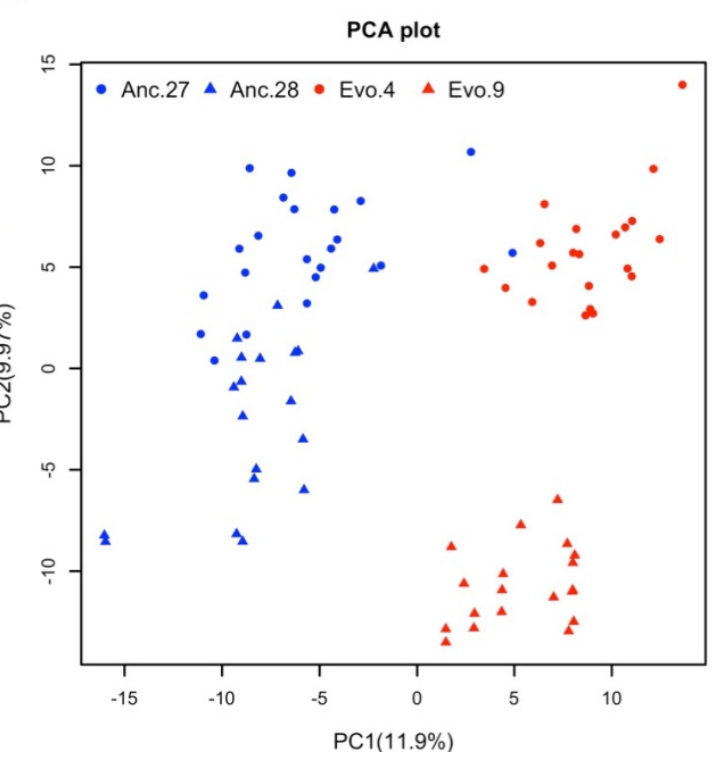

Figure 3. Schematic overview of the experimental procedures (a) and the divergence in

303 from one common founder population, two replicate populations evolved for 100 generations

304 in a hot laboratory environment fluctuating between 18 and $28^{\circ} \mathrm{C}$. Common garden

305 Experiment: after 100 generations, the two evolved replicate populations were maintained

306 together with the reconstituted ancestral population for two generations in a hot laboratory

307 environment fluctuating between 18 and $28^{\circ} \mathrm{C}$. After this common garden procedure, about

30830 males from each population were subjected to RNA-Seq. b. Principle Component

309 Analysis (PCA) of the transcriptomic profiles of individuals from the ancestral population

310 (blue) and the hot-evolved population (red). Circles indicate individuals of the first replicate

311 (Anc. No. 27 and Evo. No. 4). Triangles represent individuals of the second replicate (Anc.

312 No. 28 and Evo. No. 9). The two replicates were made with two different batches of library

313 cards for RNA-Seq library preparation. 
a

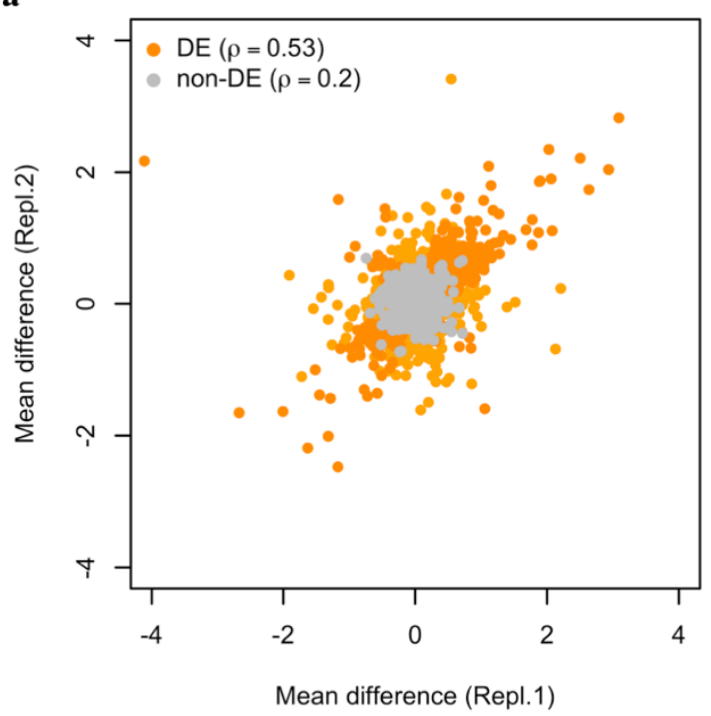

c

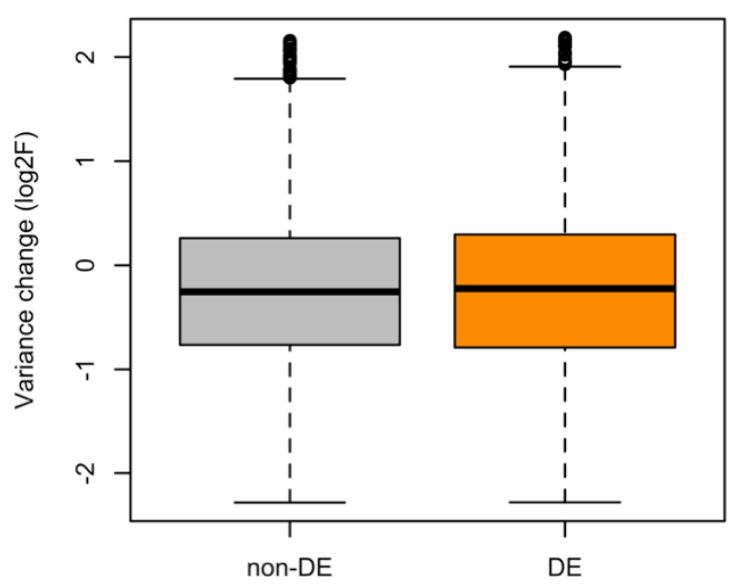

b

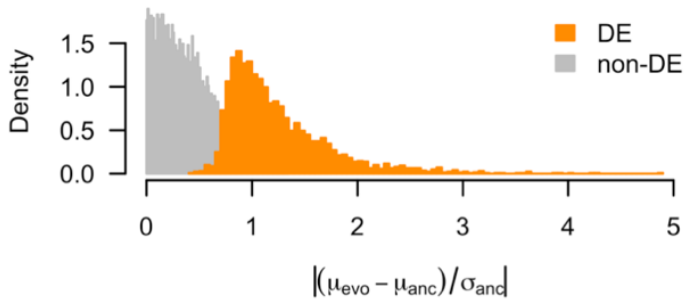

Replicate2

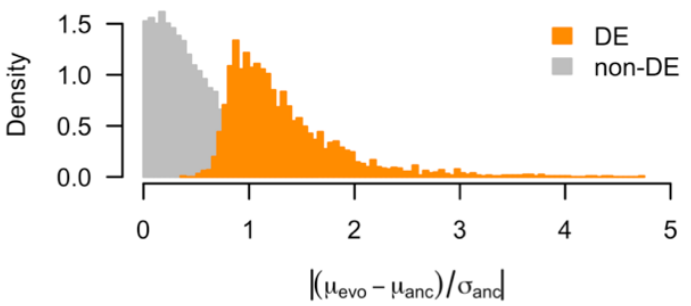

Replicate2

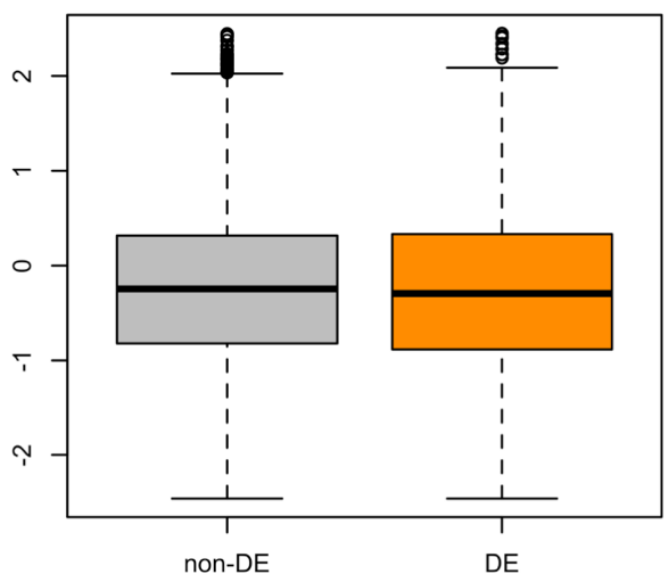

316 Figure 4. Evolution of phenotypic mean and variance over 100 generations of

317 adaptation in empirical data. a. The evolution of gene expression mean during adaptation

318 in the two replicates. For the genes with significant changes (DE, in orange), the changes are

319 correlated between replicates (Spearman's rho $=0.53$ ). For the genes without significant

320 changes (non-DE, in grey), the correlation between replicates is much lower (Spearman's rho

$321=0.2)$. b. The evolution of gene expression mean scaled by the ancestral variation. For the

322 DE genes (in orange), the median change falls around 1 standard deviation of the ancestral expression value, suggesting mild shift in trait optimum in the novel environment. For the 
324 non-DE genes (in grey), the changes in expression are mostly negligible. The same pattern is

325 seen in the second replicate. c. The change in expression variance during adaptation for DE

326 and non-DE genes. In both replicates, the distribution of variance changes is

327 indistinguishable between DE genes (orange) and non-DE genes (grey) (Wilcoxon's rank

328 sum test, $\mathrm{p}>0.1$ for both replicates).

329 
Materials and Methods

\section{Computer simulations}

332 We performed forward simulations with MimicrEE2 (Vlachos and Kofler, 2018) using the

333 qff mode to illustrate the influence of the genetic architecture on the evolution of phenotypic

334 variance during the adaptation to a new trait optimum. With 189 founder haplotypes (Barghi et al., 2019), we simulated quantitative traits under the control of different numbers of loci (5, $25,50,100,200$ and 1000) with an effective population size of 300 . For each trait, we assume an additive model and a negative correlation $(\mathrm{r}=-0.7$, estimated in Barghi et al., 2019) between the effect size and starting frequency (Figure 1b). The effect sizes of each locus can disperse in different levels which depend on the shape parameter of gamma sampling process (shape $=0.5,2.5$ and 100, Figure 1c). We used correlate() function implemented in "fabricatr" R package (Blair et al., 2019) to generate the effect sizes. The sum of effect sizes of each trait was normalized to 1 . We assumed a heritability $\mathrm{h}^{2}=0.6$ (from a family-based estimation in this study). To simulate stabilizing selection with trait optimum shift, we provided the Guassian fitness functions with mean of $\overline{X_{\text {anc. }}}+a \sqrt{V_{\text {anc }}}$. and standard deviation of $b \sqrt{V_{a n c}}$, where $\overline{X_{a n c}}$. is the ancestral phenotypic mean and $V_{\text {anc. }}$ is the ancestral genetic variance (Figure 1a). Parameter "a" determines the distance of

347 optimum shift, which is set to one (similar to the empirical case, Figure 4b) or three (Adopted 348 from Sella et al., 2019). Parameter "b" indicates how strong the phenotypic constrain would 349 be when the trait optimum is reached. The value 3.6 is adopted from Sella et al., 2019. In this study, we increase and decrease it by $50 \%$ to explore its impact (1.8 or 5.4$)$. For the neutrality case, we assumed uniform fitness for all individuals. For each trait under each scenario, the phenotypic variance was estimated at different generations and compared to the ancestral phenotypic variance at generation 1 to illustrate the dynamic of phenotypic variance during the evolution. We note that we do not assume that the ancestral population has reached an 
equilibrium, because the ancestral population in this study was phenotyped in the new environment.

Experimental evolution

359 The setup of populations and evolution experiment have been described by (Barghi et al., 360 2019). Briefly, ten outbred populations seeded from 202 isofemale lines were exposed to a 361 laboratory experiment at $28 / 18{ }^{\circ} \mathrm{C}$ with $12 \mathrm{hr}$ light/12hr dark photoperiod for more than 100 generations. Each replicate consisted of 1000 to 1250 adults at each generation.

\section{Common garden experiment}

365 The collection of samples from the evolution experiment for RNA-Seq was preceded by two generations of common garden (CGE). The common garden experiment was performed at generation 103 of the evolution in the hot environment and this CGE has been described in (Barghi et al., 2019; Hsu et al., 2020, 2019; Jakšić et al., 2020). In brief, an ancestral population was reconstituted by pooling five mated females from 184 founder isofemale lines

370 (Nouhaud et al., 2016). No significant allele frequency differences are expected between the reconstituted ancestral populations and the original ancestral populations initiating the experiment (Nouhaud et al., 2016). Because we evaluated phenotypes on the population level, deleterious mutations will have a very limited impact. The reason is that they occur only in a single isofemale line, which represents a very small fraction of the total population.

375 Two replicates of the reconstituted ancestral population and two independently evolved populations at generation 103 were reared for two generations with egg-density control (400 eggs/bottle) at the same temperature regime as in the evolution experiment. Freshly eclosed

378 flies were transferred onto new food for mating. Sexes were separated under $\mathrm{CO}_{2}$ anesthesia at day 3 after eclosure, left to recover from $\mathrm{CO}_{2}$ for two days, and at the age of five days 
whole-body mated flies of each sex were snap-frozen at $2 \mathrm{pm}$ in liquid nitrogen and stored at $-80^{\circ} \mathrm{C}$ until RNA extraction. In this study, more than 30 individual male flies from two reconstituted ancestral populations (replicate no. 27 and no. 28) and two evolved populations (replicate no. 4 and no. 9) were subjected to RNA-Seq.

$\underline{\text { RNA extraction and library preparation }}$

Whole bodies of individual male flies were removed from the $-80^{\circ} \mathrm{C}$ freezer and immediately homogenized in Qiazol lysis reagent (Qiagen, Hilden, Germany). The homogenate was treated with DNase I followed by addition of chloroform, centrifugation and mixture of the upper phase with 70\% ethanol as described for the Qiagen RNeasy Universal Plus Mini Kit.

390 The mixture was subsequently loaded onto a RNeasy MinElute Spin column as provided by the RNeasy Plus Micro Kit (Qiagen, Hilden, Germany), and all washing steps were performed according to the instructions for that kit. All resulting total RNA was used to prepare stranded mRNA libraries on the Neoprep Library Prep System (Illumina, San Diego, USA) following the manufacturer's protocol: Neoprep runs were performed using software version 1.1.0.8 and protocol version 1.1.7.6 with default settings for 15 PCR cycles and an insert size of $200 \mathrm{bp}$. All libraries for individuals of ancestral replicate no. 27 and evolved replicate no. 4 were prepared with library cards of lot no. 20180170; all libraries for individuals of ancestral replicate no. 28 and evolved replicate no. 9 were prepared with library cards of lot no. 20178099. 50bp single-end reads were sequenced on an Illumina

400 HiSeq 2500. All sequencing data will be available in European Nucleotide Archive (ENA) under the accession number PRJEB37011 upon publication. 
404 All RNA-Seq reads were trimmed using ReadTools (Gómez-Sánchez and Schlötterer, 2018)

405 with quality score of 20 and aligned to Drosophila simulans reference genome (Palmieri et

406 al., 2015) using GSNAP (Wu et al., 2016) with parameter setting -k 15 -N 1 -m 0.08.

407 Exon-aligned reads were piped into Rsubread (Liao et al., 2019) to calculate read counts of

408 each gene, and raw read counts of each gene were normalized with the TMM method

409 implemented in edgeR (Robinson et al., 2010). Samples with severe 3'- bias were removed

410 based on visual inspection of the gene-body coverage plot (Jakšić and Schlötterer, 2016;

411 Wang et al., 2012).

412

413 Genetic variance in gene expression across F1 families

414 We evaluated how much of the expression variance can be explained by genetic variation by

415 performing RNA-Seq on individual flies, with 3-4 individuals each from three isofemale lines

416 maintained at the same density and culturing conditions.

417 Six out of 184 founder isofemale lines from the evolution experiment and were maintained

418 for one generation with controlled egg density (400 eggs/bottle) in the same environment as

419 the main experiment $\left(12 \mathrm{~h} 28^{\circ} \mathrm{C}\right.$ with light followed by $12 \mathrm{~h} 18{ }^{\circ} \mathrm{C}$ with dark conditions).

420 Using the offspring, we generated three crosses between two of the six lines each: FL $138 \mathrm{x}$

421 FL 137, FL 157 x FL 112, FL 123 x FL 127: we combined 50 virgin females from one of the

422 lines with 50 males from the other line, let them lay eggs under density control as above and

423 maintained and froze their F1 offspring in the same way as in the main CGE: sexes were 424 separated after mating at the age of three days and snap-frozen at the age of five days at $2 \mathrm{pm}$.

425 From each cross, we used four F1 males to prepare individual RNA-Seq libraries as described 426 above. 
427 Assuming no environmental heterogeneity, we decomposed the total variance of the

428 expression of each gene measured in these individuals into the genetic difference among

429 three different F1 families and random error. The data were analyzed as follows:

430 Natural log-transformation was applied to CPMs of all genes to improve data normality

431 (Rocke and Durbin, 2003). Principal component and principal variance component analysis

432 (Bushel, 2019) was performed to the whole transcriptome to decompose the variance

433 components. We found that around $60 \%$ of the gene expression variance can be explained by

434 the genetic difference among the three F1 families (method - Figure supplement 1a and b).

435 This implies that the within-population gene expression variance is largely contributed by the

436 genetic components. Because we only used offspring from single vials, we may have

437 overestimated the heritability if the environment in the vials differs. Nevertheless, since our

438 heritability estimates are very similar to previous ones (Ayroles et al., 2009), we consider our

439 estimates reliable.

440

441 In addition to general analysis across all genes, we also tested for the genetic variance of each

442 gene separately using analysis of variance (ANOVA):

$$
y_{i j}=\mu+\tau_{i}+\varepsilon_{i j}
$$

444 Where $\mathrm{i}=1,2,3, \ldots, \mathrm{n}$ (the $\mathrm{i}^{\text {th }}$ genes) $\mathrm{j}=1,2,3,4$ (the $\mathrm{j}^{\text {th }}$ individuals in each cross). $y_{i j}$ is the 445 observed expression level of a gene in a given sample, $\mu$ is the overall mean; $\tau_{i}$ is the 446 effect of genetic background and $\varepsilon_{i j}$ is the random noise. We calculated the proportion of

447 total variance explained by random error using the following equation:

$$
\text { variance explained by random error }=\frac{\text { sum squares of error }(S S E)}{\text { sum squares of total }(S S T)}
$$


449 Genes were binned based on their average expression value (lnCPM) which ranged from -0.8

450 to 4.1 , by bin size of 0.1 . The average proportion of variance explained by random error of

451 each bin was calculated.

452 The expression variance of genes with less than 1 count per million bases (CPM) is 453 dominated by technical errors (method - Figure supplement 1c). Thus, genes with less than 4541 count per million base (CPM) were excluded for subsequent analysis.

\section{$\underline{\text { RNA-Seq data analysis }}$}

457 We observed some outlier individuals and suspected that the freezing process may have led to detachment of body parts, such as eyes or heads, in these individuals. We compared gene expression between such outliers and all other samples and performed tissue enrichment analysis for genes with at least 2-fold lower expression in the outlier samples. Samples with evidence of tissue detachment were excluded. After filtering, each population remained approximately 20 individuals (Supplementary file 1). Only genes with at least 1 count per million base (CPM) were included in the analyses to avoid extremely lowly expressed genes.

464 For all RNA-Seq data we only compared samples which were prepared with library cards 465 from the same lot number to avoid batch effects (Replicate 1: evolved replicate 4 vs. 466 reconstituted ancestral population replicate 27; Replicate 2: evolved replicate 9 ss.

467 reconstituted ancestral population replicate 28$)$.

468 For differential expression analysis on mean expression, we used the generalized linear 469 modeling function implemented in edgeR (Robinson et al., 2010) to fit the expression to the 470 model $(Y=E+\varepsilon)$ in which $\mathrm{Y}$ stands for gene expression, $\mathrm{E}$ is the effect of evolution and $\varepsilon$ 471 is the random error. The likelihood ratio test was performed to test the effect of evolution.

472 P-value adjustment was performed using the Benjamini-Hochberg false discovery rate (FDR) 473 correction. 
474 For the analysis of expression variance evolution, we applied natural log transformation

475 (Rocke and Durbin, 2003) to eliminate the strong mean-variance dependency in RNA-Seq

476 data due to the nature of the negative binomial distribution (method - Figure supplement 2).

477 The variance of the expression of each gene (lnCPM) was estimated in each population. With

478 the moderate sample size, we needed to estimate the uncertainty of variance estimates.

479 Jackknifing was applied to measure the uncertainty of estimator (Fukunaga and Hummels,

480 1989). The procedure was conducted independently on four replicates and we calculated the

$48195 \%$ confidence interval of the estimated variance (method - Figure supplement 3 ). The

482 change of gene expression variance was determined by the F statistics calculated as the ratio

483 between the variance within the ancestral population and the variance within the evolved

484 population of each gene. To test whether selection alter the expression variance, a

485 comparison was made between the F statistics of genes with significant changes in mean

486 expression and the ones without.

487 
Acknowledgments

489 Special thanks to David Houle, who provided fantastic support during the collection and

490 establishment of the isofemale lines in Florida. We thank all member of the Institut für

491 Populationsgenetik for discussion. We are grateful to Reinhard Bürger and David Houle for

492 helpful comments on earlier versions of the manuscript. Neda Barghi, François Mallard and

493 Kathrin Otte contributed to the common garden experiment. Illumina sequencing was

494 performed at the VBCF NGS Unit (www.vbcf.ac.at). This work was support by the Austrian

495 Science Funds (FWF, W1225) and the European Research Council (ERC, ArchAdapt).

496

497 Author contribution

498 W.Y.L and C.S. conceived the study. V.N. prepared all RNA-Seq and supervised the

499 maintenance of the evolution experiment. A.M.J supervised the common garden experiment.

500 W.Y.L performed the simulation and data analysis. W.Y.L. and C.S. wrote the manuscript.

501

502 Competing interests

503 The authors declare no competing interests.

504

505 Correspondence and requests for materials should be addressed to C.S.

506 


\section{References}

509

510

511

512

513

514

515

516

517

518

Ayroles JF, Carbone MA, Stone EA, Jordan KW, Lyman RF, Magwire MM, Rollmann SM, Duncan LH, Lawrence F, Anholt RRH, Mackay TFC. 2009. Systems genetics of complex traits in Drosophila melanogaster. Nat Genet 41:299-307. doi:10.1038/ng.332

Barghi N, Hermisson J, Schlötterer C. 2020. Polygenic adaptation: a unifying framework to understand positive selection. Nat Rev Genet 21:769-781. doi: $10.1038 / \mathrm{s} 41576-020-0250-\mathrm{Z}$

Barghi N, Tobler R, Nolte V, Jakšić AM, Mallard F, Otte KA, Dolezal M, Taus T, Kofler R, Schlötterer C. 2019. Genetic redundancy fuels polygenic adaptation in Drosophila. PLOS Biol 17:e3000128. doi:10.1371/journal.pbio.3000128

Barton NH, Etheridge AM, Véber A. 2017. The infinitesimal model: Definition, derivation, and implications. Theor Popul Biol 118:50-73. doi:10.1016/J.TPB.2017.06.001

Barton NH, Keightley PD. 2002. Understanding quantitative genetic variation. Nat Rev Genet 3:11-21. doi:10.1038/nrg700

Barton NH, Turelli M. 1987. Adaptive landscapes, genetic distance and the evolution of quantitative characters. Genet Res 49:157-173. doi:10.1017/S0016672300026951

Boyle EA, Li YI, Pritchard JK. 2017. An expanded view of complex traits: From polygenic to omnigenic. Cell 169:1177-1186. doi:10.1016/J.CELL.2017.05.038

Bulmer MG. 1972. The genetic variability of polygenic characters under optimizing selection, mutation and drift. Genet Res 19:17-25. doi:10.1017/S0016672300014221

Burke MK, Dunham JP, Shahrestani P, Thornton KR, Rose MR, Long AD. 2010. Genome-wide analysis of a long-term evolution experiment with Drosophila. Nature 467:587-590. doi:10.1038/nature09352

Bushel P. 2019. pvca: Principal variance component analysis (PVCA). $R$ Packag version 
533 Castle WE. 1921. An improved method of estimating the number of genetic factors concerned in the cases of blending inheritance. Science 54:223. doi:10.1126/science.54.1393.223

536 Chevalet C. 1994. An approximate theory of selection assuming a finite number of quantitative trait loci. Genet Sel Evol. 26:379. doi:10.1186/1297-9686-26-5-379

Chippindale AK, Chu TJF, Rose MR. 1996. Complex trade-offs and the evolution of starvation resistance in Drosophila melanogaster. Evolution (N Y) 50:753-766. doi:10.1111/j.1558-5646.1996.tb03885.x

Clutton-Brock TH, Pemberton JM (Josephine M). 2004. Soay sheep : population dynamics and selection on St. Kilda. Cambridge University Press.

Daborn PJ, Yen JL, Bogwitz MR, Le Goff G, Feil E, Jeffers S, Tijet N, Perry T, Heckel D, Batterham P, Feyereisen R, Wilson TG, ffrench-Constant RH. 2002. A single p450 allele associated with insecticide resistance in Drosophila. Science 297:2253-6. doi:10.1126/science. 1074170

Endler L, Gibert J-M, Nolte V, Schlötterer C. 2018. Pleiotropic effects of regulatory variation in tan result in correlation of two pigmentation traits in Drosophila melanogaster. Mol Ecol 27:3207-3218. doi:10.1111/mec.14781

Falconer DS, Mackay TFC. 1963. Introduction to quantitative genetics, Poultry Science. CreateSpace Independent Publishing Platform. doi:10.3382/ps.0420547

Ferea TL, Botstein D, Brown PO, Rosenzweig RF. 1999. Systematic changes in gene expression patterns following adaptive evolution in yeast. Proc Natl Acad Sci USA 96:9721-6. doi:10.1073/pnas.96.17.9721

Franssen SU, Kofler R, Schlötterer C. 2017. Uncovering the genetic signature of quantitative trait evolution with replicated time series data. Heredity (Edinb) 118:42-51. 
Fukunaga K, Hummels DM. 1989. Leave-one-out procedures for nonparametric error estimates. IEEE Trans Pattern Anal Mach Intell 11:421-423. doi:10.1109/34.19039

Gómez-Sánchez

$\mathrm{D}$, Schlötterer C. 2018. ReadTools: A universal toolkit for handling sequence data from different sequencing platforms. Mol Ecol Resour 18:676-680. doi:10.1111/1755-0998.12741

Hayward LK, Sella G. 2019. Polygenic adaptation after a sudden change in environment. bioRxiv 792952. doi:10.1101/792952

Hsu S-K, Jakšić AM, Nolte V, Barghi N, Mallard F, Otte KA, Schlötterer C, Hsu S-K, Jakšić AM, Nolte V, Barghi N, Mallard F, Otte KA, Schlötterer C. 2019. A 24 h age difference causes twice as much gene expression divergence as 100 generations of adaptation to a novel environment. Genes (Basel) 10:89. doi:10.3390/genes10020089

Hsu S-K, Jakšić AM, Nolte V, Lirakis M, Kofler R, Barghi N, Versace E, Schlötterer C. 2020. Rapid sex-specific adaptation to high temperature in Drosophila. Elife 9. doi:10.7554/eLife.53237

Huang W, Carbone MA, Magwire MM, Peiffer JA, Lyman RF, Stone EA, Anholt RRH, Mackay TFC. 2015. Genetic basis of transcriptome diversity in Drosophila melanogaster. Proc Natl Acad Sci $U$ S $\quad$ A 112:E6010-E6019. doi:10.1073/pnas. 1519159112

Huang Y, Agrawal AF. 2016. Experimental evolution of gene expression and plasticity in

PLOS

Genet

12:e1006336.

Jain K, Stephan W. 2015. Response of polygenic traits under stabilizing selection and doi:10.1534/g3.115.017970 
Jakšić AM, Karner J, Nolte V, Hsu S-K, Barghi N, Mallard F, Otte KA, Svečnjak L, Senti K-A, Schlötterer C. 2020. Neuronal function and dopamine signaling evolve at high temperature in Drosophila. Mol Biol Evol 37:2630-2640. doi:10.1093/molbev/msaal 16

Jakšić AM, Schlötterer C. 2016. The interplay of temperature and genotype on patterns of alternative splicing in Drosophila melanogaster. Genetics

204:315-325. doi:10.1534/GENETICS.116.192310

Kawecki TJ, Lenski RE, Ebert D, Hollis B, Olivieri I, Whitlock MC. 2012. Experimental evolution. Trends Ecol Evol 27:547-560. doi:10.1016/J.TREE.2012.06.001

Keightley PD, Hill WG. 1989. Quantitative genetic variability maintained by mutation-stabilizing selection balance: sampling variation and response to subsequent directional selection. Genet Res 54:45-58. doi:10.1017/S0016672300028366

Kimura M, Crow JF. 1964. The number of alleles that can be maintained in a finite population. Genetics 49:725-738.

Lai W.-Y. \& Schlötterer, C. 2021. Evolution of gene expression variance during adaptation to high temperature in Drosophila. BioRxiv.

Lande R. 1976. Natural selection and random genetic drift in phenotypic evoltion. Evolution (N Y) 30:314-334. doi:10.1111/j.1558-5646.1976.tb00911.x

Lenski RE, Travisano M, Larison B, Moritz C. 1994. Dynamics of adaptation and diversification: a 10,000-generation experiment with bacterial populations. Proc Natl Acad Sci 91:6808-6814. doi:10.1073/pnas.91.15.6808

Liao Y, Smyth GK, Shi W. 2019. The R package Rsubread is easier, faster, cheaper and better for alignment and quantification of RNA sequencing reads. Nucleic Acids Res 47:e47-e47. doi:10.1093/nar/gkz114

Liu X, Li YI, Pritchard JK. 2019. Trans effects on gene expression can drive omnigenic inheritance. Cell 177:1022-1034.e6. doi:10.1016/J.CELL.2019.04.014 
Mackay TFC, Stone EA, Ayroles JF. 2009. The genetics of quantitative traits: challenges and

608 prospects. Nat Rev Genet 10:565-577. doi:10.1038/nrg2612

609

Mallard F, Nolte V, Tobler R, Kapun M, Schlötterer C. 2018. A simple genetic basis of adaptation to a novel thermal environment results in complex metabolic rewiring in Drosophila. Genome Biol 19:119. doi:10.1186/s13059-018-1503-4

612 Nielsen R. 2005. Molecular signatures of natural selection. Annu Rev Genet 39:197-218. doi:10.1146/annurev.genet.39.073003.112420

614 Nouhaud P, Tobler R, Nolte V, Schlötterer C. 2016. Ancestral population reconstitution from isofemale lines as a tool for experimental evolution. Ecol Evol 6:7169-7175. doi:10.1002/ece3.2402

Otte KA, Nolte V, Mallard F, Schlötterer C. 2020. The adaptive architecture is shaped by population ancestry and not by selection regime. bioRxiv 2020.06.25.170878. doi: $10.1101 / 2020.06 .25 .170878$

Palmieri N, Nolte V, Chen J, Schlötterer C. 2015. Genome assembly and annotation of a Drosophila simulans strain from Madagascar. Mol Ecol Resour 15:372-81.

Pélabon C, Hansen TF, Carter AJR, Houle D. 2010. Evolution of variation and variability under fluctuating, stabilizing, and disruptive selection. Evolution (N Y) 64:1912-25. doi:10.1111/j.1558-5646.2010.00979.x

Pritchard JK, Pickrell JK, Coop G. 2010. The genetics of human adaptation: Hard sweeps, soft sweeps, and polygenic adaptation.

Curr Biol

20:R208-R215. doi:10.1016/J.CUB.2009.11.055

Robinson MD, McCarthy DJ, Smyth GK. 2010. edgeR: a Bioconductor package for differential expression analysis of digital gene expression data. Bioinformatics 26:139140. doi:10.1093/bioinformatics/btp616 
632 Rocke DM, Durbin B. 2003. Approximate variance-stabilizing transformations for gene-expression microarray data. Bioinformatics 19:966-72.

634 Romero IG, Ruvinsky I, Gilad Y. 2012. Comparative studies of gene expression and the evolution of gene regulation. Nat Rev Genet 13:505-516. doi:10.1038/nrg3229

Signor SA, Nuzhdin S V. 2018. The evolution of gene expression in cis and trans. Trends Genet 34:532-544. doi:10.1016/j.tig.2018.03.007

Sork VL. 2017. Genomic studies of local adaptation in natural plant populations. J Hered 109:3-15. doi:10.1093/jhered/esx091

Suvorov A, Nolte V, Pandey RV, Franssen SU, Futschik A, Schlötterer C. 2013. Intra-specific regulatory variation in Drosophila pseudoobscura. PLoS One 8:e83547. doi:10.1371/journal.pone.0083547

Turelli M. 1984. Heritable genetic variation via mutation-selection balance: Lerch's zeta meets the abdominal bristle. Theor Popul Biol 25:138-193.

Wang L, Wang S, Li W. 2012. RSeQC: quality control of RNA-seq experiments. Bioinformatics 28:2184-2185. doi:10.1093/bioinformatics/bts356

648 Wittkopp PJ, Haerum BK, Clark AG. 2008. Regulatory changes underlying expression differences within and between Drosophila species. Nat Genet 40:346-350. doi:10.1038/ng. 77

Wittkopp PJ, Haerum BK, Clark AG. 2004. Evolutionary changes in cis and trans gene regulation. Nature 430:85-88. doi:10.1038/nature02698

$653 \mathrm{Wu}$ TD, Reeder J, Lawrence M, Becker G, Brauer MJ. 2016. GMAP and GSNAP for genomic sequence alignment: Enhancements to speed, accuracy, and functionality. Humana Press, New York, NY. pp. 283-334. doi:10.1007/978-1-4939-3578-9_15

656 Yoo BH, Nicholas FW, Rathie KA. 1980. Long-term selection for a quantitative character in 
bioRxiv preprint doi: https://doi.org/10.1101/2021.01.19.427260; this version posted January 20, 2021. The copyright holder for this preprint (which was not certified by peer review) is the author/funder, who has granted bioRxiv a license to display the preprint in perpetuity. It is made available under aCC-BY-NC-ND 4.0 International license. 
661

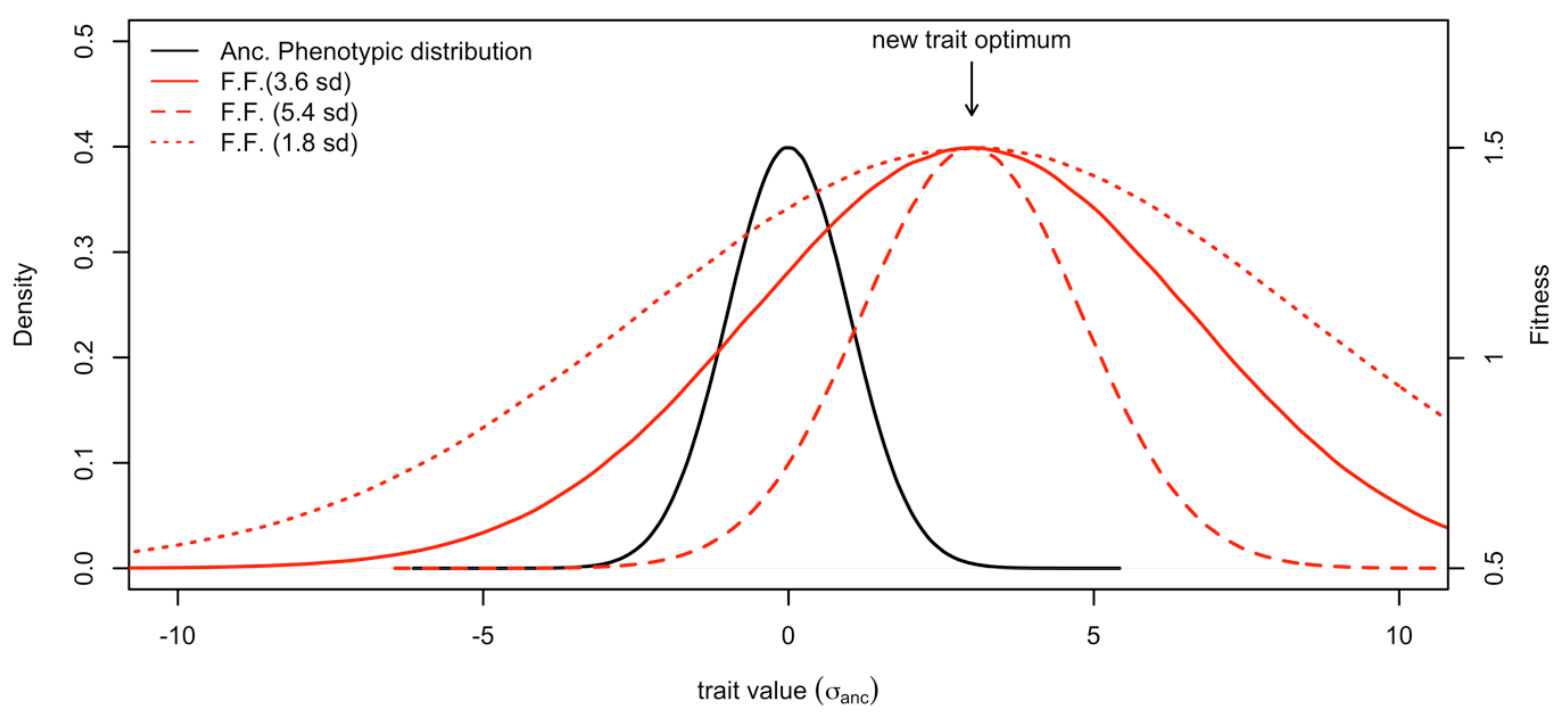

662 Figure 1 - Figure supplement 1. The evolutionary scenario for distant optimum shift.

663 We consider the case when a quantitative trait (in black) experiences a sudden shift in trait

664 optimum under stabilizing selection. The imposed fitness functions (F.F.) are illustrated in

665 red. The new trait optimum is set away from the ancestral trait mean by three standard

666 deviation of the ancestral trait distribution for distal shift. To vary the strength of stabilizing

667 selection, the variance of the fitness function is set as 1.8, 3.6 and 5.4 standard deviation of

668 the ancestral trait distribution.

669 


\section{Distant optimum shift $\left(3 \sigma_{\text {anc }}\right)$}

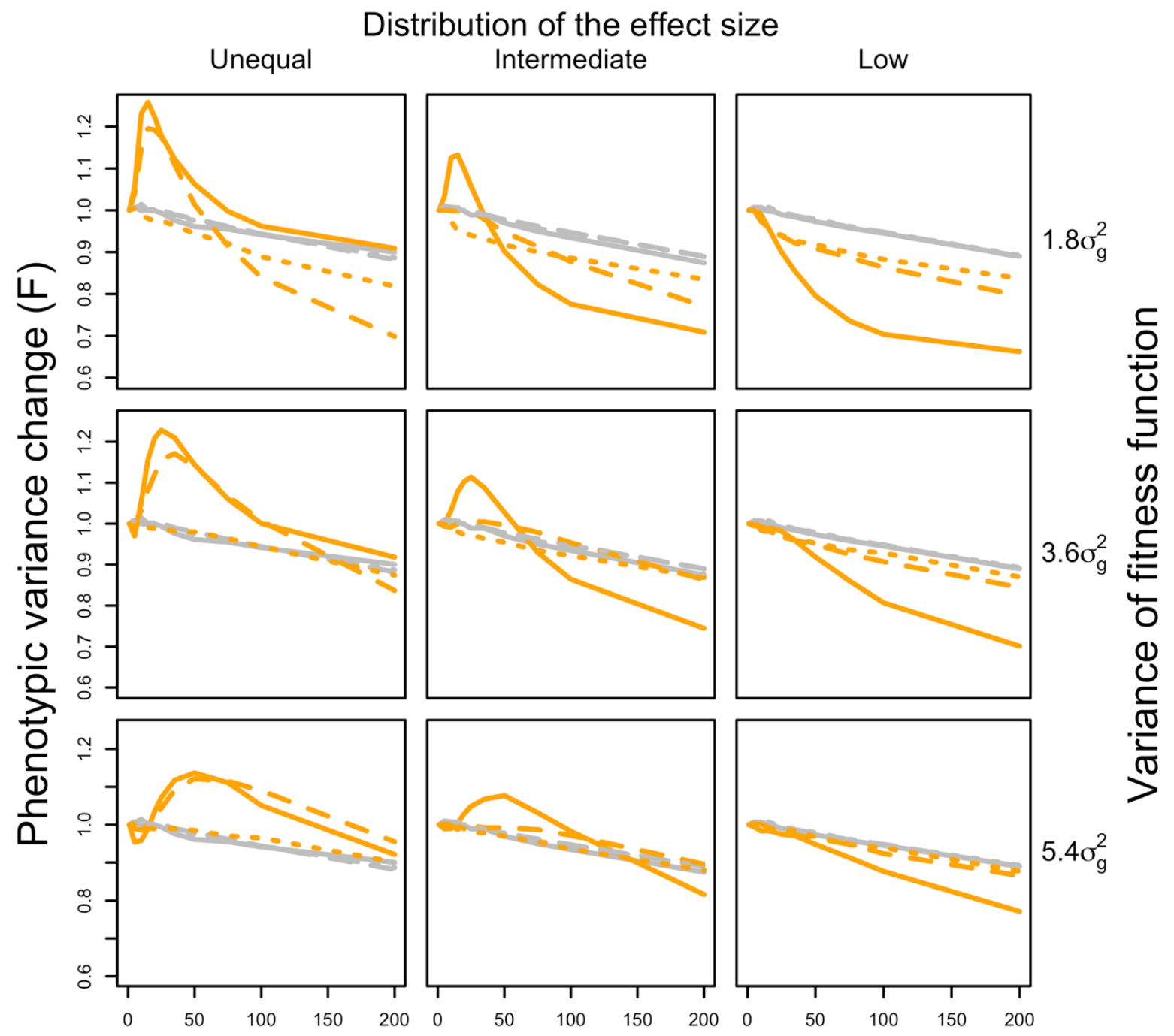

\section{Generation}

672 Figure 2 - Figure supplement 1. The trajectory of expected changes in phenotypic

673 variance when adapting to a distant optimum shift. The changes in phenotypic variance

674 within 200 generations adapting to a distant optimum shift (orange) are compared to the

675 changes under neutrality (grey) on y axis. The change in variance $(\mathrm{F})$ is calculated as the ratio

676 of phenotypic variance between each evolved time point (generation $\mathrm{x}$ ) and the ancestral state

$677\left(\sigma_{200}^{2} / \sigma_{0}^{2}\right)$. The simulations cover traits controlled by varying numbers of loci underlying the

678 adaptation with three different distributions of effect sizes (columns) and under different 
679 strength of stabilizing selection (rows). For each scenario, 1000 runs of simulations have

680 been performed. Only traits with the most (dotted lines, 1000 loci), intermediate (dash lines,

68150 loci) and the least (solid lines, 5 loci) polygenic architectures are shown. Unlike the

682 continuous decreasing pattern in the cases with moderate optimum shifts, the variance of

683 traits controlled by a few loci (5 loci) with largely dispersed effects would increase first and

684 then decrease when the effect sizes of contributing loci are dispersed (orange solid lines).

685 Nevertheless, for traits with extremely polygenic basis, the phenotypic variance always stays

686 stable over time (orange dotted lines).

687 


\section{Mild optimum shift ( $\left.1 \sigma_{\text {anc }}\right)$}

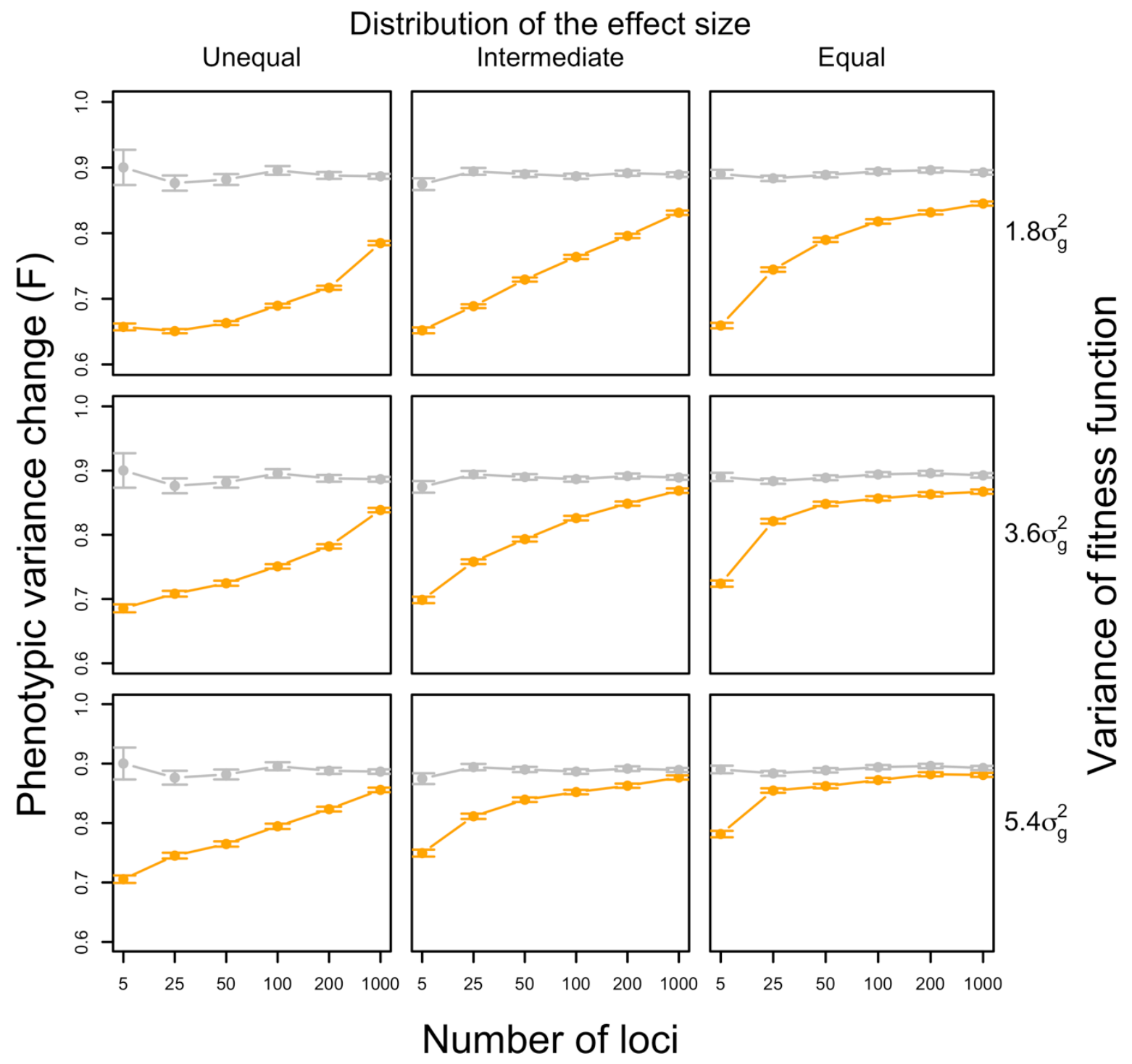

688

689 Figure 2 - Figure supplement 2. The expected changes in phenotypic variance and the

690 number of contributing loci. The changes in phenotypic variance after 200 generations

691 adapting to a mild optimum shift (orange) are compared to the changes under neutrality (grey)

692 on y axis. The change in variance $(\mathrm{F})$ is calculated as the ratio between the evolved and

693 ancestral phenotypic variance $\left(\sigma_{200}^{2} / \sigma_{0}^{2}\right)$. The simulations cover traits controlled by varying

694 numbers of loci underlying the adaptation (x-axes) with three different distributions of effect

695 sizes (columns) and under different strength of stabilizing selection (rows). For each scenario,

6961000 runs of simulations have been performed. In all scenarios, the variance of the trait 
697 decreases drastically when the adaptation is controlled by a small number of loci. As the

698 number of contributing loci increases, the phenotypic variance becomes more stable.

699 


\section{Distant optimum shift $\left(3 \sigma_{\text {anc }}\right)$}

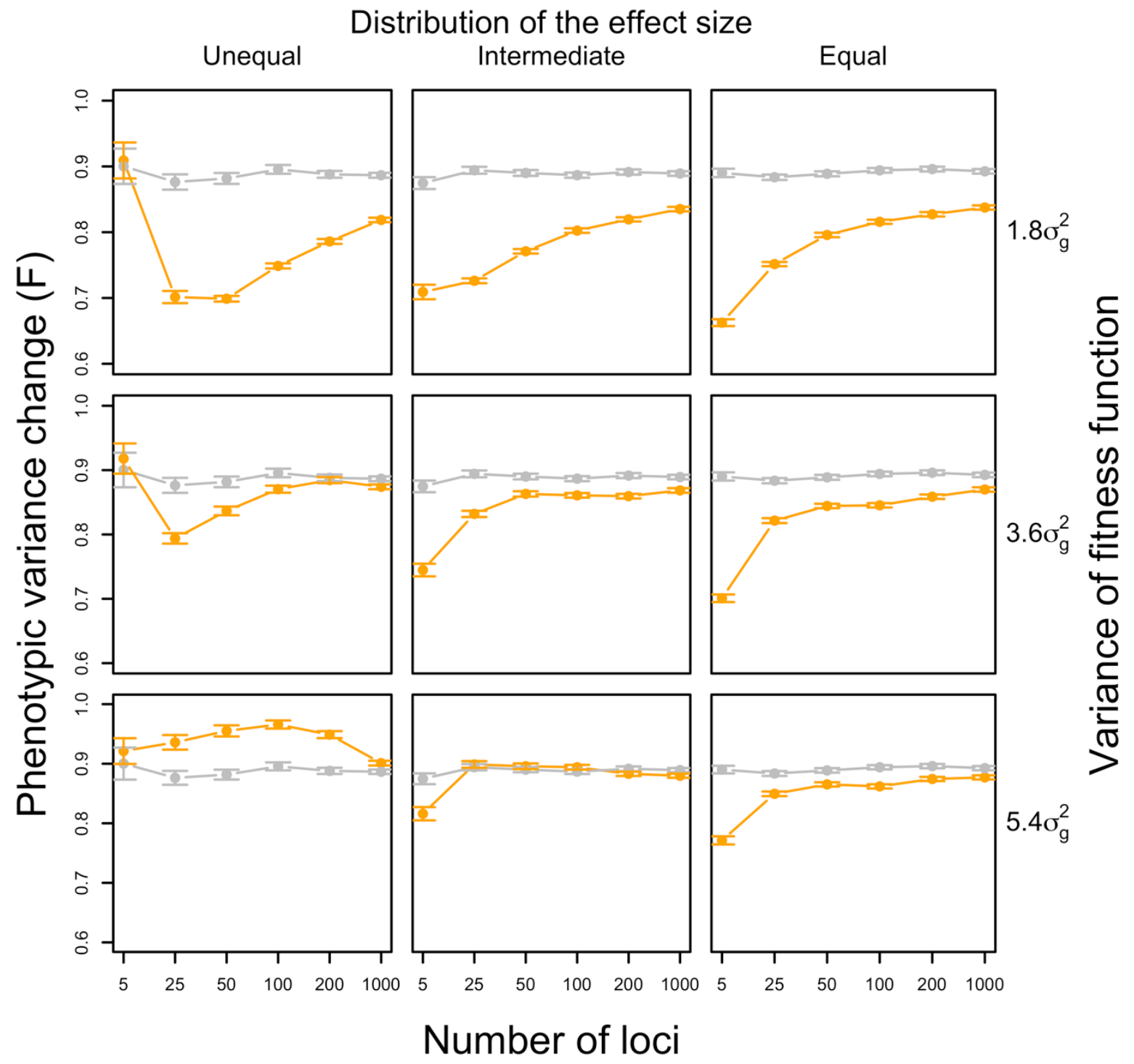

Figure 2 - Figure supplement 3. The expected changes in phenotypic variance and the

number of contributing loci. The changes in phenotypic variance after 200 generations

adapting to a distant optimum shift (orange) are compared to the changes under neutrality

704 (grey) on y axis. The change in variance $(\mathrm{F})$ is calculated as the ratio between the evolved and ancestral phenotypic variance $\left(\sigma_{200}^{2} / \sigma_{0}^{2}\right)$. The simulations cover traits controlled by varying numbers of loci underlying the adaptation (x-axes) with three different distributions of effect sizes (columns) and under different strength of stabilizing selection (rows). For each scenario, 1000 runs of simulations have been performed. In most scenarios, the variance of 
709 the trait decreases drastically when the adaptation is controlled by a small number of loci. As

710 the number of contributing loci increases, the phenotypic variance becomes more stable.

711 However, exceptions can be observed when the effect sizes of the contributing loci are

712 largely dispersed. In these cases, shift in optimum does not have the strong impact on the

713 variance of traits under simple genetic control (5 contributing loci). In the extreme case,

714 highly dispersed effect sizes in combination with a relaxed phenotypic constraint removes the

715 relationship between the number of loci and the changes in phenotypic variance.

716 


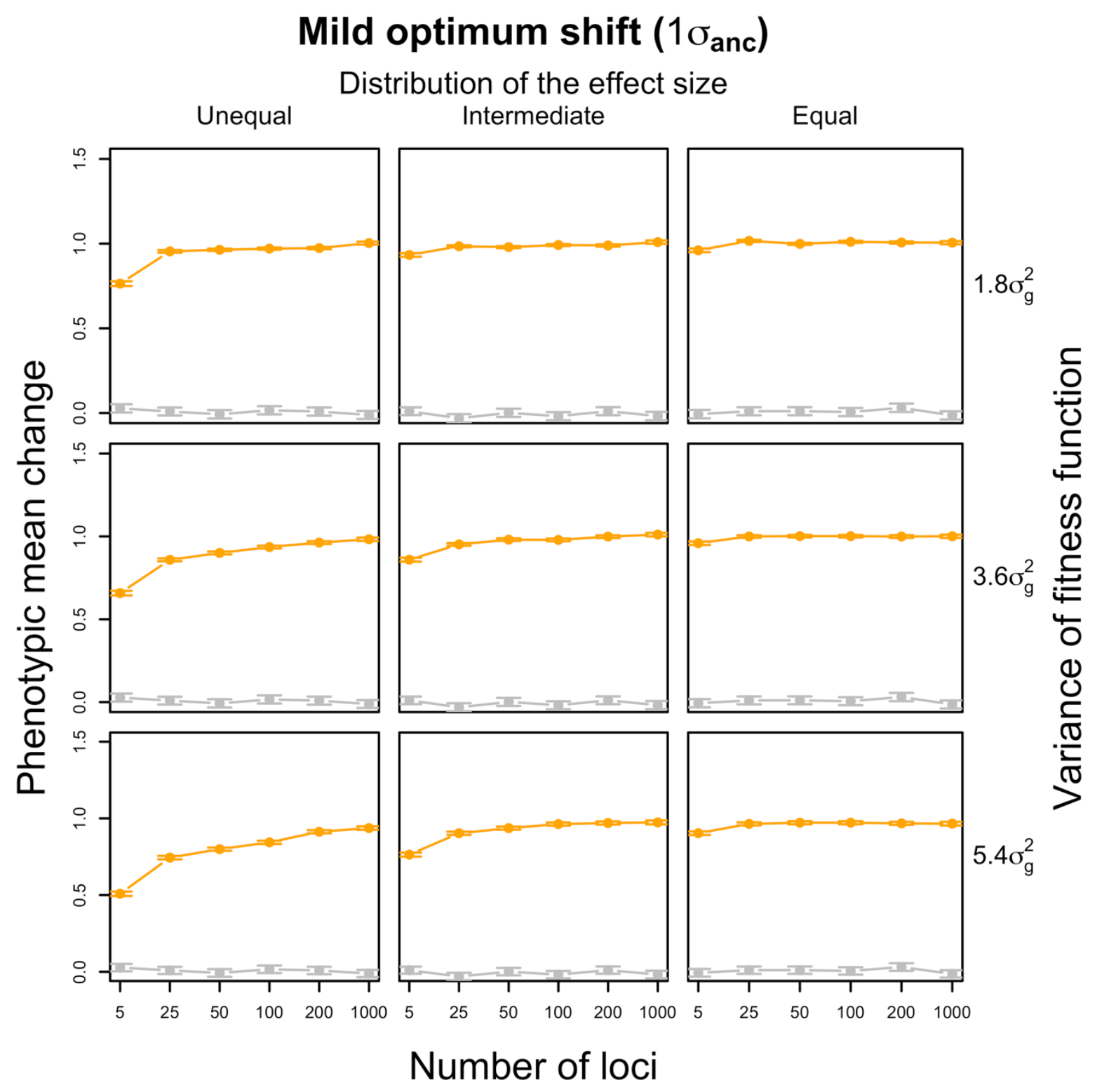

719 Figure 2 - Figure supplement 4. The changes in phenotypic mean when adapting to a

mild optimum shift. The changes in phenotypic mean after 200 generations adapting to a

721 mild optimum shift (orange) are compared to the changes under neutrality (grey) on y axis.

722 The changes in phenotypic mean are scaled by the standard deviation of the ancestral trait

723 distribution. The simulations cover traits controlled by varying numbers of loci underlying

724 the adaptation (x axes) with three different distributions of effect sizes (columns) and under different strength of stabilizing selection (rows). For each scenario, 1000 runs of simulations 
726 have been performed. In most cases, the traits under selection (orange) shift their means by

727 one standard deviation of the ancestral trait distribution (i.e. reaching the new trait optimum)

728 while the neutral traits (grey) stay unchanged. 


\section{Distant optimum shift $\left(3 \sigma_{\text {anc }}\right)$}

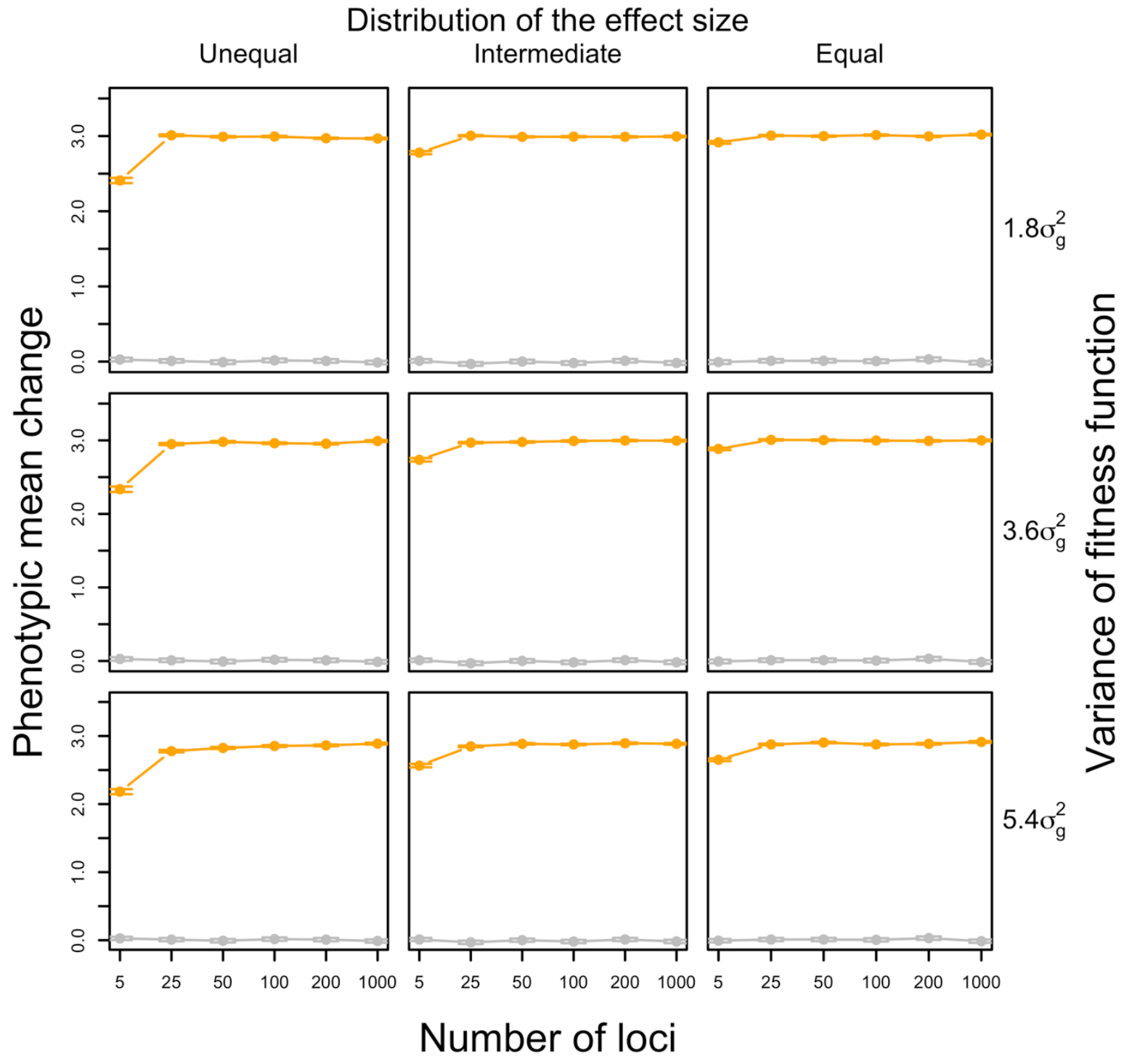

731 Figure 2 - Figure supplement 5. The changes in phenotypic mean when adapting to a

732 distant optimum shift. The changes in phenotypic mean after 200 generations adapting to a

733 distant optimum shift (orange) are compared to the changes under neutrality (grey) on y axis.

734 The changes in phenotypic mean are scaled by the standard deviation of the ancestral trait

735 distribution. The simulations cover traits controlled by varying numbers of loci underlying

736 the adaptation (x axes) with three different distributions of effect sizes (columns) and under

737 different strength of stabilizing selection (rows). For each scenario, 1000 runs of simulations

738 have been performed. In most cases, the traits under selection (orange) shift their means by 
bioRxiv preprint doi: https://doi.org/10.1101/2021.01.19.427260; this version posted January 20, 2021. The copyright holder for this preprint (which was not certified by peer review) is the author/funder, who has granted bioRxiv a license to display the preprint in perpetuity. It is made available under aCC-BY-NC-ND 4.0 International license.

739 three standard deviation of the ancestral trait distribution (i.e. reaching the new trait optimum)

740 while the neutral traits (grey) stay unchanged.

741 

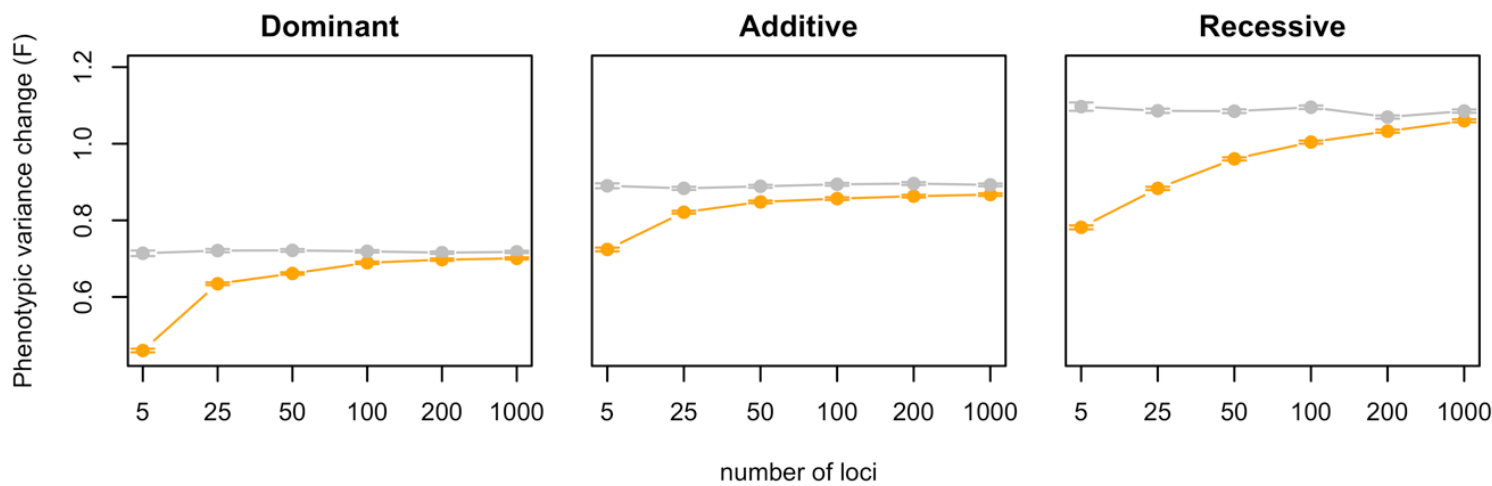

743 Figure 2 - Figure supplement 6. The expected changes in phenotypic variance for traits

744 controlled by dominant and recessive alleles. The changes in phenotypic variance after 200

745 generations adapting to a mild optimum shift (orange) are compared to the changes under

746 neutrality (grey) on y axis. The change in variance $(\mathrm{F})$ is calculated as the ratio between the

747 evolved and ancestral phenotypic variance $\left(\sigma_{200}^{2} / \sigma_{0}^{2}\right)$. This simulation covers traits controlled

748 by varying numbers of loci underlying the adaptation ( $\mathrm{x}$ axes) with recessive, additive and

749 dominant effects. For each scenario, 1000 runs of simulations have been performed. No

750 matter how the dominance varies, the variance of the trait decreases drastically when the

751 adaptation is controlled by a small number of loci. As the number of contributing loci

752 increases, the phenotypic variance becomes more stable. 


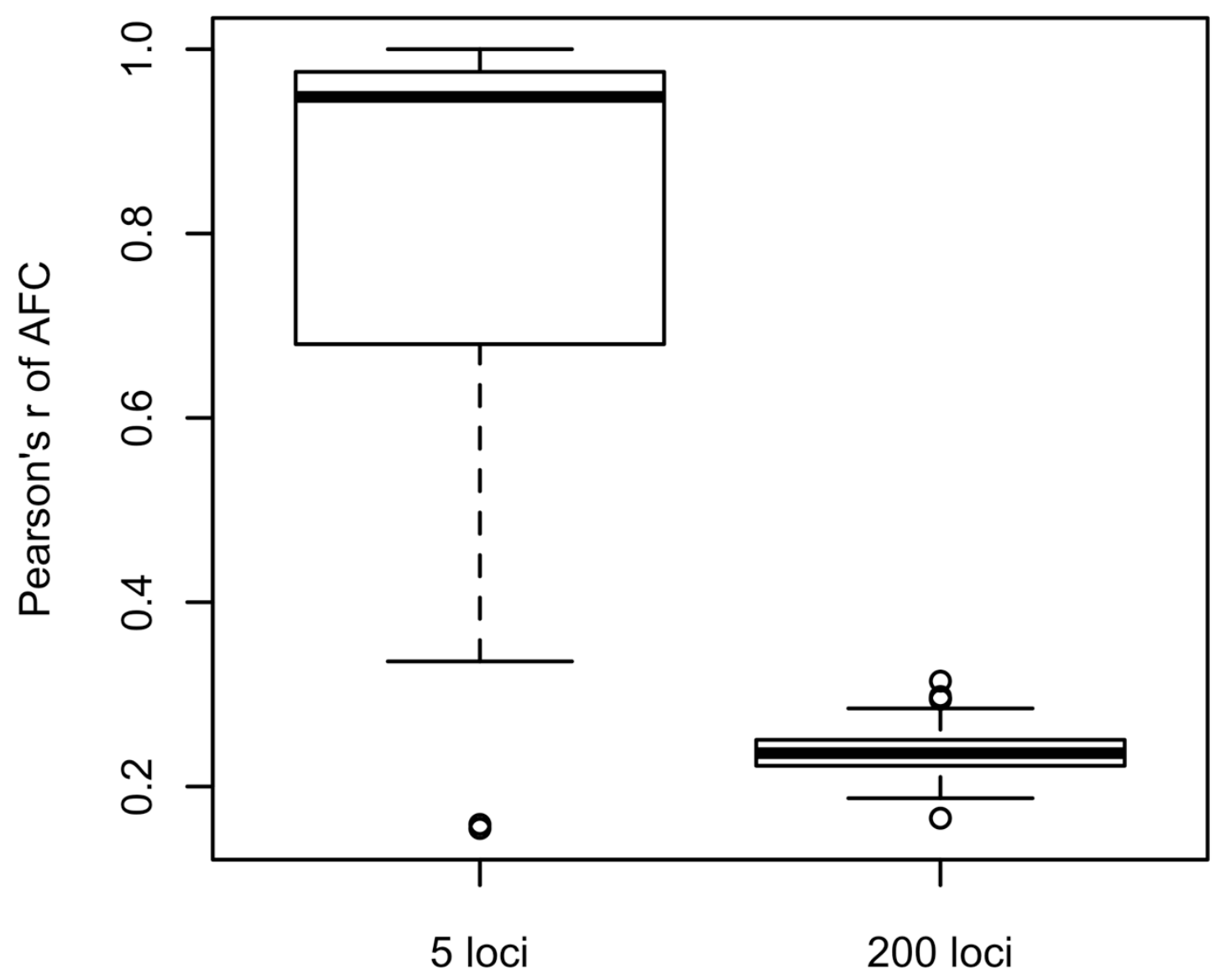

755 Figure 2 - Figure supplement 7. Parallelism in genomic response across 10 evolved

756 replicates for traits under the control of five loci and $\mathbf{1 0 0}$ loci. For the loci with allele

757 frequency change of at least $10 \%$ in 200 generations, the average Pearson's correlation

758 coefficient of the frequency change between all pairs of two evolved replicates was

759 calculated to describe the parallelism of the evolution at these loci. An average across loci is

760 used to obtain a general parallelism. 100 runs of simulations with 10 evolution replicates

761 have been performed for each scenario. With five contributing loci, the genomic evolution of 
762 the contributing loci is more parallel across the 10 evolved replicates compared to the case

763 when with 100 contributing loci.

764 
(A)

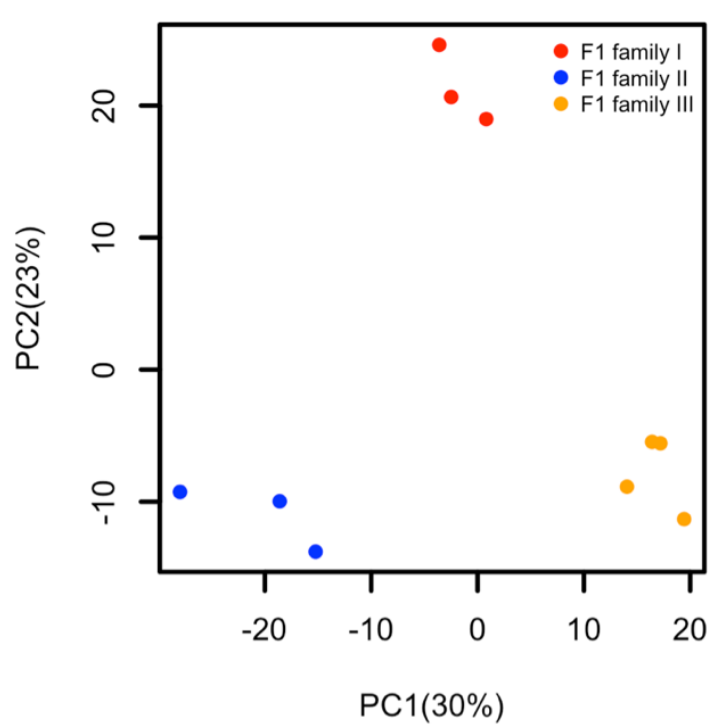

(B)

\section{PVCA estimation bar chart}

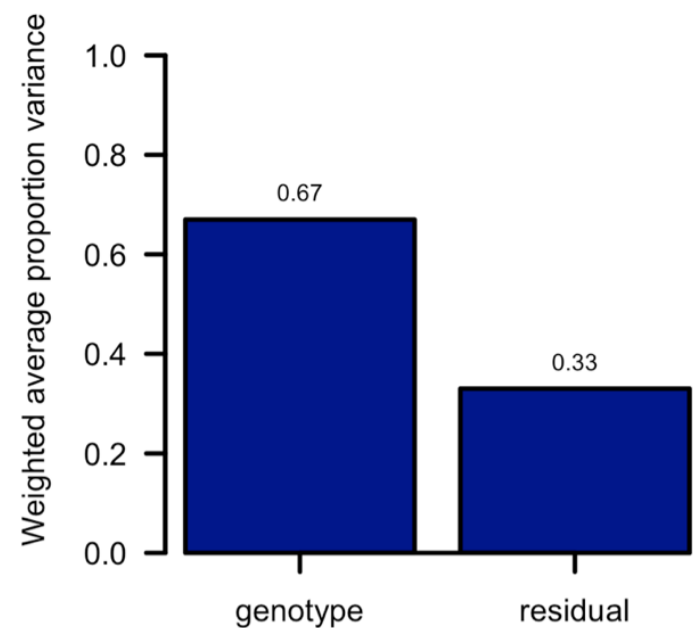

Effects

(C)

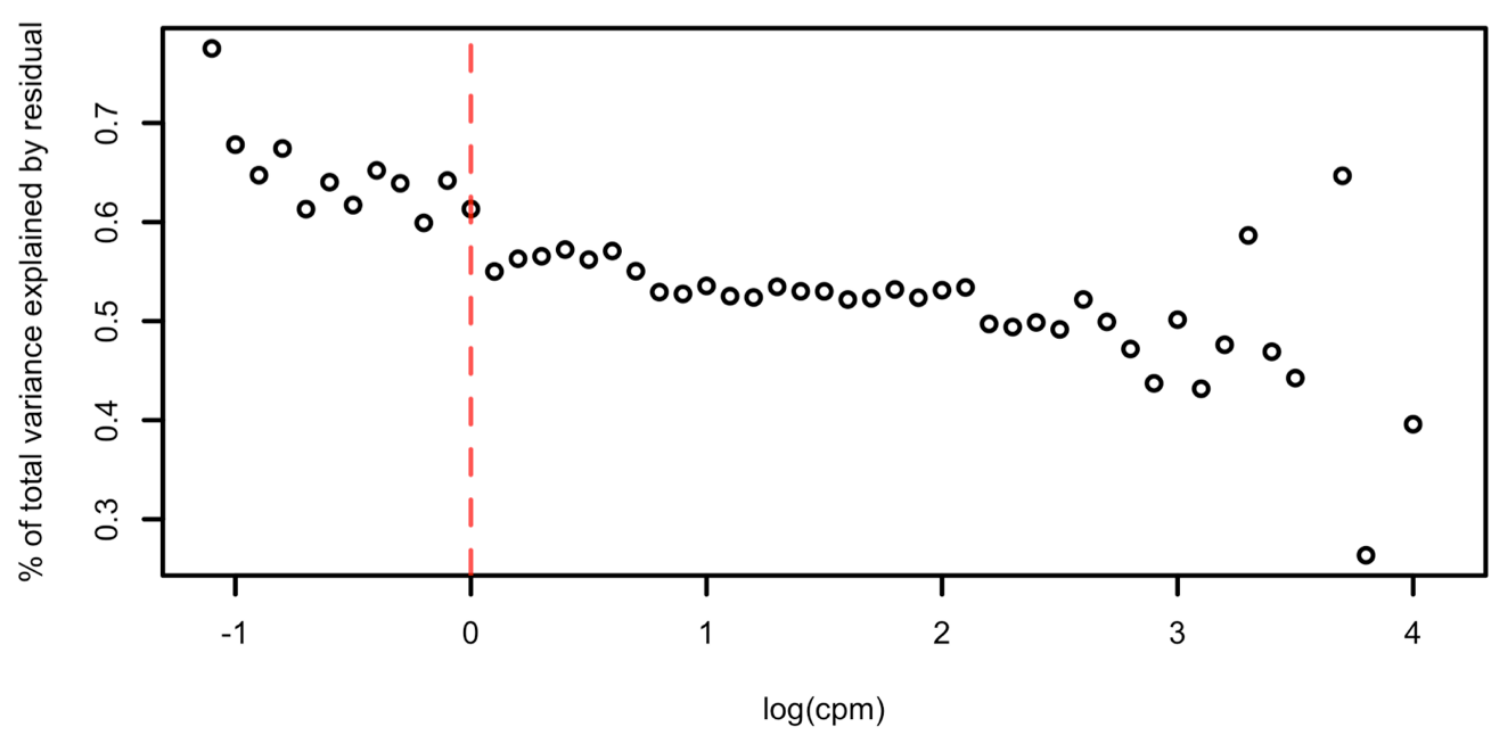

766 Method - Figure supplement 1. Genetic variance in gene expression across F1 families.

767 (A) Principal component analysis (PCA) on the transcriptomes of F1 individuals from three

768 different crosses between the founder iso-female lines. Individuals from different families

769 clustered nicely based on the first two PCs. (B) Principal variance component analysis

770 (PVCA) on the transcriptomes of $\mathrm{F} 1$ individuals. 67\% of the total variance in gene expression

771 was explained by the genetic difference between the individuals. (C) Gene-wise analysis of 
772 variance (ANOVA) in gene expression. Genes were binned based on their average expression

773 value (lnCPM) which ranged from -0.8 to 4.1 , by bin size of 0.1 . The average proportion of

774 variance explained by random error of each bin was visualized. The expression variance of

775 genes with less than 1 count per million bases (CPM) is dominated by residuals.

776 
(A)

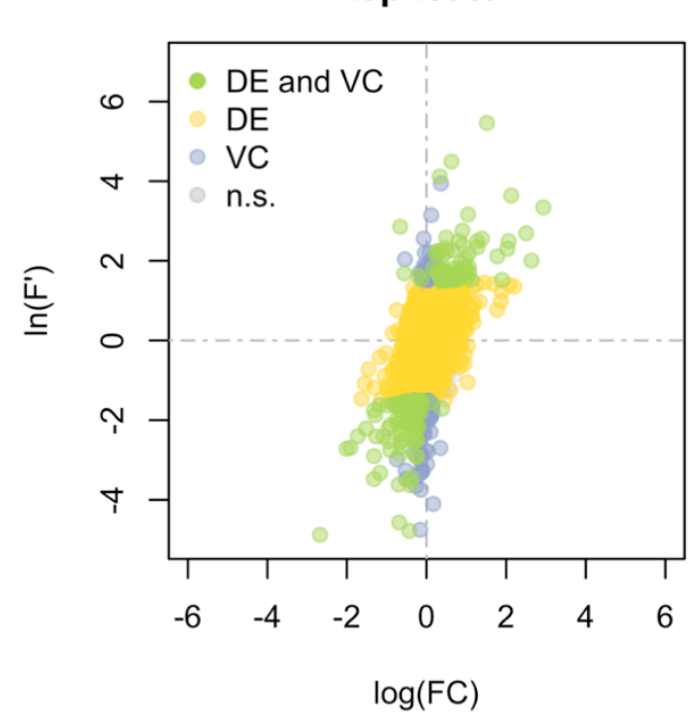

(B)

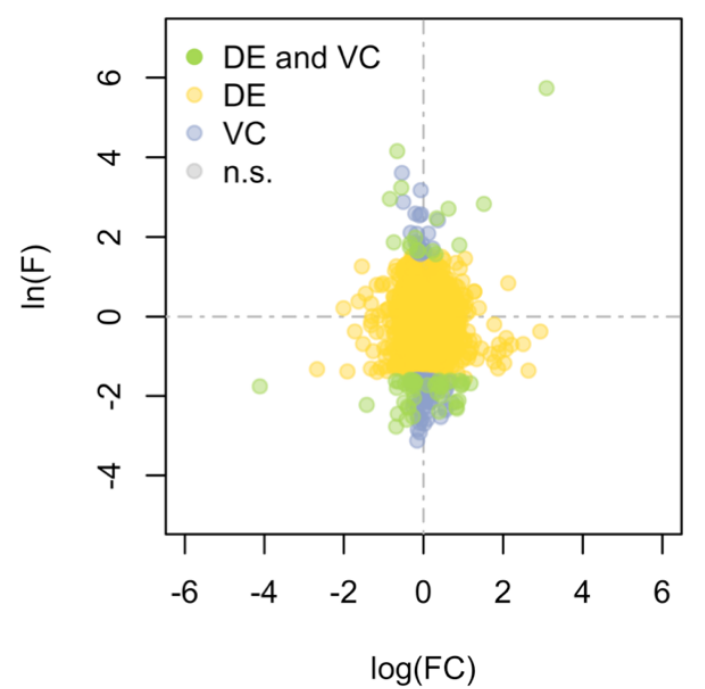

\section{Replicate1}

\section{Replicate2}

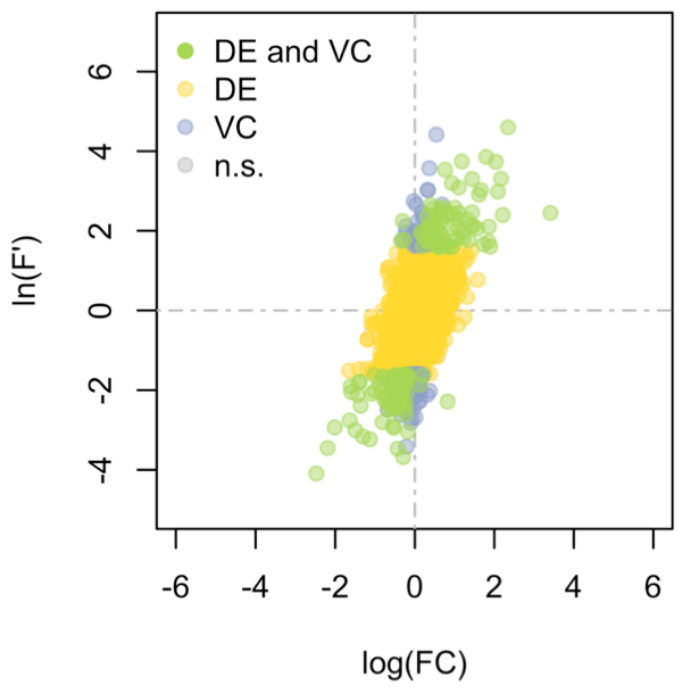

Replicate2

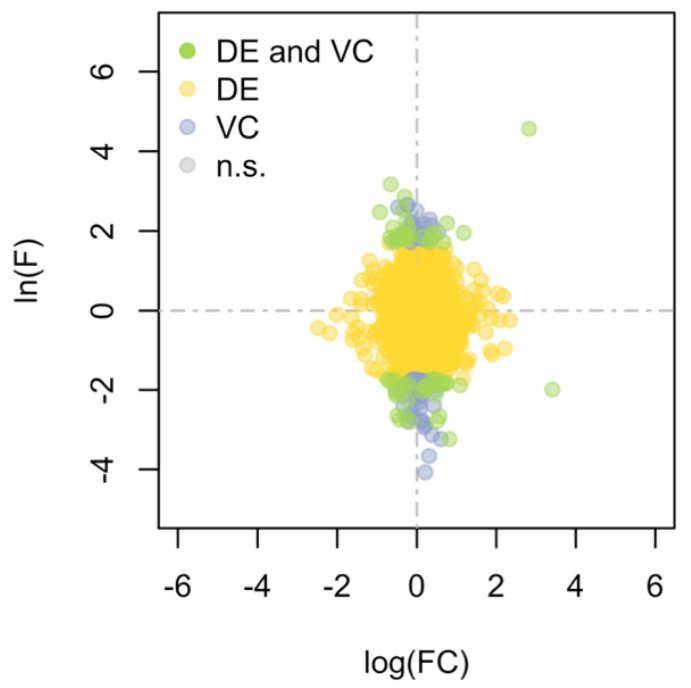

\section{Method - Figure supplement 2. Log-transformation eliminates the positive relationship}

between the changes in mean and variance of gene expression. In each panel, the changes in mean expression, $\log (\mathrm{FC})\left(F C=\frac{\overline{y_{\text {evo. }}}}{\overline{y_{\text {anc. }}}}\right)$ and in variance before $(\mathbf{A})$ and after $(\mathbf{B})$ the natural $\log$-transformation of each gene were visualized $\left(F^{\prime}=\frac{\operatorname{var}\left(y_{\text {evo. }}\right)}{\operatorname{var}\left(y_{\text {anc. }}\right)}\right.$ and $\left.F=\frac{\operatorname{var}\left(\ln \left(y_{\text {evo. }}\right)\right)}{\operatorname{var}\left(\ln \left(y_{\text {anc. }}\right)\right)}\right)$.

782 The positive correlation $(r=0.45)$ due to the positive mean-variance dependency of negative

783 binomial distribution is removed by the log-transformation $(r=-0.05)$ on gene expression 784 level. 

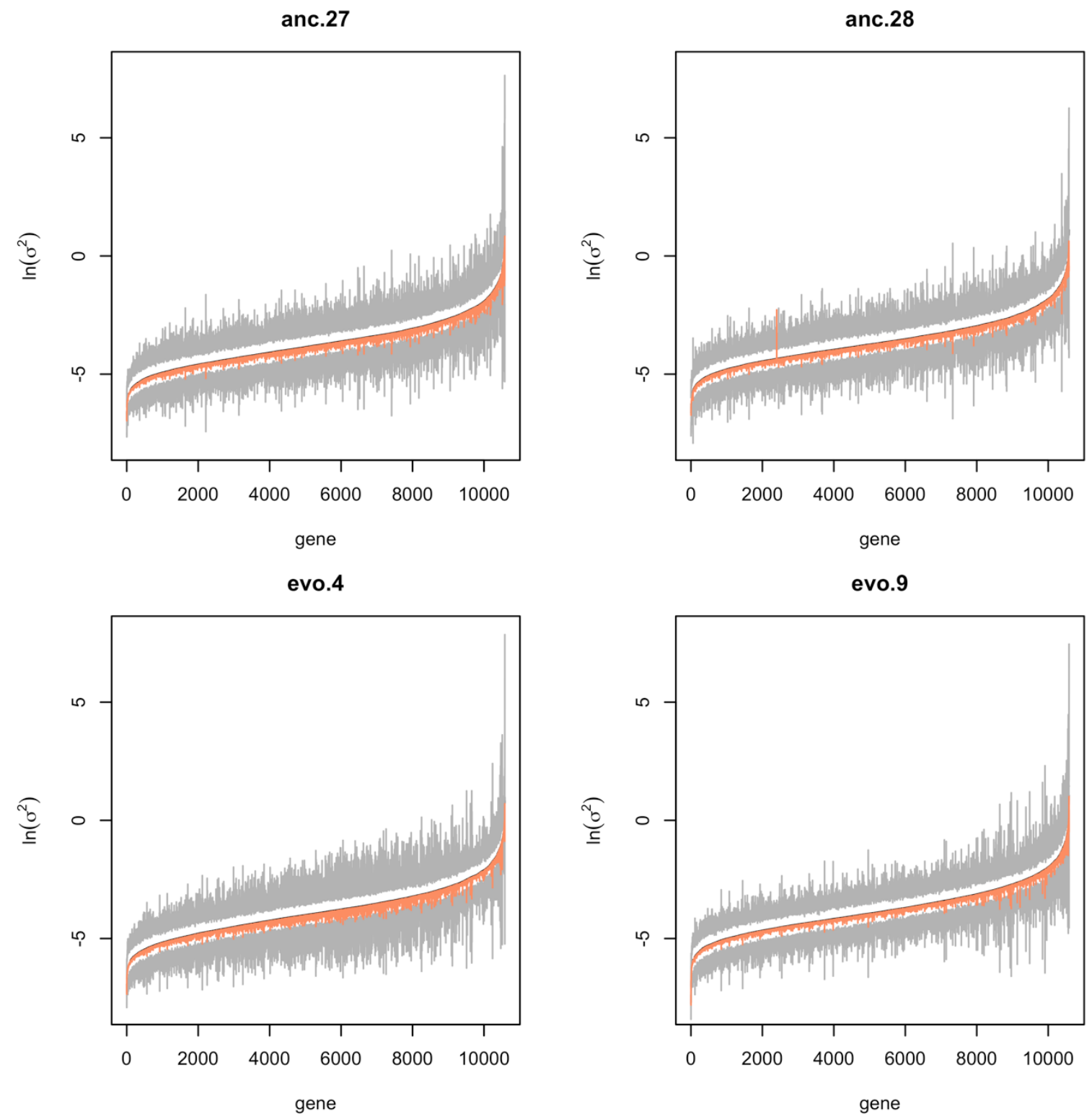

787 Method - Figure supplement 3. Robustness of the variance estimation using individual

788 sequencing data. Jackknife method was applied to measure the uncertainty of variance estimation. Given a sample size of $\mathrm{K}$, the procedure is to estimate the variance of each gene

790 for K times, each time leaving one sample out. The procedure was conducted independently

791 on 4 populations (anc.27, anc.28, evo.4 and evo.9). In each panel, we visualize Jackknife 792 approximated 95\% confidence interval for the variance estimates of each gene. The genes are 793 ordered based on the average variance estimates (black dash line) on the x-axis. The upper and lower limits of the $95 \%$ confidence interval are indicated with grey curves. The salmon 
795 line denotes the observed value of the variance estimates. In most cases, the estimates lie in

796 the confidence interval, suggesting robust estimation.

797 
798 Titles and legend for supplementary files

799 Supplementary file 1. Library information of the sample in this study. This file provides

800 a list of all sequenced samples and the library information.

801

802 Supplementary file 2. Differential gene expression analysis of two contrasts between

803 ancestral and evolved populations. This file reports the results of DE analysis between anc.

80427 and evo. 4 (Table S2A) and between anc. 28 and evo. 9 (Table S2B).

805

806 Supplementary file 3. F value on the gene expression of two contrasts between ancestral

807 and evolved populations. This file reports the results of gene expression variance

808 comparisons between anc. 27 and evo. 4 (Table S3A) and between anc. 28 and evo. 9 (Table

809 S3B).

810 